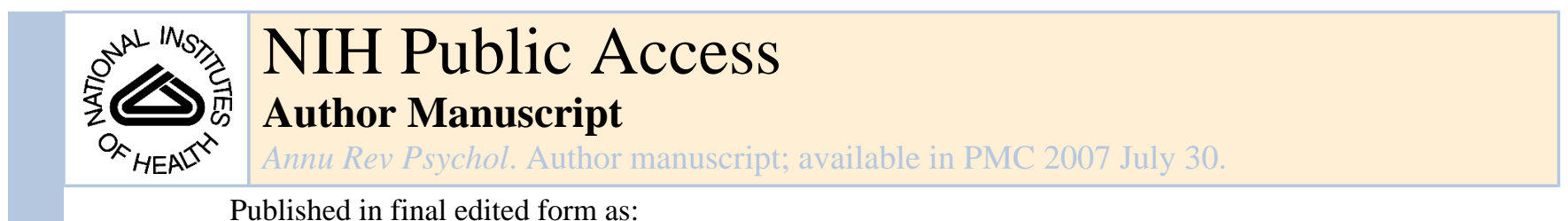

Published in final edited form as:

Aпnи Rev Psychol. 2007 ; 58: 373-403.

\title{
The Experience of Emotion
}

\author{
Lisa Feldman Barrett ${ }^{1}$, Batja Mesquita ${ }^{2}$, Kevin N. Ochsner ${ }^{3}$, and James J. Gross ${ }^{4}$ \\ 1Department of Psychology, Boston College, Chestnut Hill, Massachusetts 02467 and Psychiatric \\ Neuroimaging Research Program, Massachusetts General Hospital, Harvard Medical School, Charlestown, \\ Massachusetts 02129; email: barretli@bc.edu
}

2Department of Psychology, Wake Forest University, Winston-Salem, North Carolina 27109; email: mesquita@wfu.edu

3Department of Psychology, Columbia University, New York, New York 10027; email:

kochsner@paradox.psych.columbia.edu

4Department of Psychology, Stanford University, Stanford, California 94305; email:

james@psych.stanford.edu

\begin{abstract}
Experiences of emotion are content-rich events that emerge at the level of psychological description, but must be causally constituted by neurobiological processes. This chapter outlines an emerging scientific agenda for understanding what these experiences feel like and how they arise. We review the available answers to what is felt (i.e., the content that makes up an experience of emotion) and how neurobiological processes instantiate these properties of experience. These answers are then integrated into a broad framework that describes, in psychological terms, how the experience of emotion emerges from more basic processes. We then discuss the role of such experiences in the economy of the mind and behavior.
\end{abstract}

\section{Keywords}

emotion; affect; consciousness

\section{INTRODUCTION}

As psychology transformed from the science of the mind (James 1890,Wundt 1897) into the science of behavior (Skinner 1953, Watson 1919), an important topic slipped from scientific view: the subjective experience of emotion. Recently, scientific discourse on this topic has reemerged (Barrett 2006b,Frijda 2005,Lambie \& Marcel 2002,Sabini \& Silver 2005), but the prevailing wisdom remains that "motion researchers need to figure out how to escape from the shackles of subjectivity if emotion research is to thrive" (LeDoux 2000, p. 156). Our current, impoverished understanding of emotion experience is due not only to American psychology's behaviorist legacy, but also to a view of the mind that eschews phenomenology and characterizes mental states as nothing but their causes. Consequently, knowing the causes of emotion is presumed sufficient to answer the question of what the experience is. While expedient, this scientific approach leaves out an important aspect of reality: people feel something when they experience emotion. Describing how emotion experiences are caused does not substitute for a description of what is felt, and in fact, an adequate description of what people feel is required so that scientists know what to explain in the first place. 
In this chapter, we frame an emerging scientific agenda for understanding what an experience of emotion feels like and how such feelings arise. We begin by reviewing the place of experience in existing models of emotion that largely define the mind as nothing but the processes that produce it. Next, drawing on the writings of philosopher John Searle (1992, $2000,2004)$, we argue that experiences of emotion are content-rich events that emerge at the level of psychological description, but are instantiated by neurobiological processes, and any theory of emotion experience must address both content and process. We then review the available answers to what is felt (i.e., the content that makes up an experience of emotion) and how neurobiological processes account for these properties of experience. These answers are then integrated into a broad framework that describes, in psychological terms, how the experience of emotion emerges from more basic processes. Specifically, we suggest that the experience of emotion emerges from both a continuous stream of evolving affect and conceptual processing (much like the seeing "red" is a discrete experience of color that derives from a continuous spectrum of reflected light; Barrett 2006b). Affect, perceptions of the world, and conceptual knowledge about emotion are bound together at a moment in time, producing an intentional state where affect is experienced as having been caused by some object or situation. Finally, we briefly address several insights deriving from our view that have the potential to shape a scientific agenda for the study of emotion experience.

\section{SCIENTIFIC ACCOUNTS OF EMOTION EXPERIENCE}

\section{Traditional Theories of Emotion}

Most influential scientific accounts of emotion assume that experiences of emotion-like other mental events - are entailed or instantiated by physical processes in the brain or body and thus can be explained by events in the physical world. In principle, this assumption must be correct, but materialist accounts (as they are called) often go one step further by assuming that experiences can be redefined as nothing but these causes, and therefore must be understood solely in terms of them (cf. Searle 1992,2000,2004). Materialist theories differ greatly in the specifics of how emotions are caused and manifest, but they share a common assumption that an explanation of emotion experience requires only an explication of causes or effects.

\section{Materialist theories}

theories rooted in the assumption that mental contents are caused by and therefore can be redefined as nothing but physical processes

Behaviorist models of emotion, the extreme version of this view, define experience out of existence, or at least out of bounds for the scientific study of emotion (e.g., LeDoux 1996, 2000) by characterizing emotion as nothing but behavior. In this view, understanding emotion means understanding the causes of emotional behavior. Identity approaches to emotion redefine experiences of emotion as bodily states (James 1890), or, as in basic emotion models, as activity in brain circuits, neurochemical systems, so-called affect programs, or action plans (Buck 1999;Ekman 1972,1992;Izard 1977,1993;Oatley \& Johnson-Laird 1987;Panksepp 1998;Plutchik 1980), or some combination of the body and the brain (Damasio 1994,1999). ${ }^{1}$ Functionalist approaches to emotion, such as some appraisal models, define emotion by its immediate causal relations; two experiences are of the same type (e.g., anger) if they are evoked

\footnotetext{
${ }^{1}$ Identity theories reduce mental states, such as emotion experiences, to states of the nervous system. Type-type identity theories of emotion experience (e.g., the basic emotion theories) are grounded in the assumption that for every kind of emotion experience (e.g., the experience of anger), there is only one type of neurophysiological state. Token-token identity theories of emotion experience (e.g., James $1890,1894)$ argue that every instance of emotion characterized by a distinctive feeling will be identical with a distinctive physical state (e.g., different experiences of anger will be instantiated by different neurophysiological states). The current trend in some neuroscience papers to refer to emotion as increased activity in certain brain areas relies on identity assumptions about emotion.
} 
by the same psychological situation (e.g., unpleasant, another person is to blame, controllable), are defined by the same behavioral consequences (e.g., antagonistic behavior), or some combination thereof (e.g., Arnold 1960; Frijda 1986;Lazarus 1966,1991;Leventhal \& Scherer 1987;Power 1997; Roseman et al. 1990,1996;:Scherer 1984). ${ }^{2}$

\section{Biological Naturalism}

"It is a mistake to confuse the evidence that we have about a subject matter for the subject matter itself. The subject matter of psychology is the human mind, and human behavior is evidence for the existence and features of the mind, but is not itself the mind" (Searle 2004, pp. 52-53).

Biological naturalism is a philosophical framework that stands in opposition to traditional materialist views of the mind (Searle 1992,2000,2004) and in so doing offers three novel tenets for the scientific study of emotion experience. First, an adequate account of emotion experience requires more than a specification of cause; it also requires a description of content (i.e., of what is felt) that is common to all experiences of emotion and that which distinguishes one experience from another. Descriptions of phenomenological content need not convey an experience of emotion in all its richness and complexity to have scientific utility and value (i.e., describing is not experiencing) (Edelman \& Tononi 2000). ${ }^{3}$

\section{Biological naturalism}

John Searle's approach to the mind-body problem that challenges both dualist and materialist views of the mind. Consciousness is treated as a biological phenomenon that is part of the natural world (like photosynthesis or digestion). Conscious states are defined as ontologically subjective (i.e., exist only as experienced by human or animal), content-rich (i.e., they feel like something), primarily intentional events (i.e., they are about or refer to something) that are ultimately constituted by, but not redefined as, neurobiological events

Second, content cannot be entirely reduced to its causes. The experience of emotion, like any conscious state, is a system-level property of the brain (which can be explained by neuronal activity), much as digestion is a system-level property of the gastrointestinal system (which can be explained causally by the chemistry of the body) or solidity is a property of the material world (which can be explained causally by the behavior of the molecules). To conflate these

\footnotetext{
${ }^{2}$ Appraisal models began as an attempt to account for the mentalistic aspects of emotion experience in all its variety, but several prominent theories came to view the situation as the eliciting factor that produces emotional responses and in so doing, took on functionalist assumptions that reduce the experience of emotion to its immediate causal relations. Functionalist approaches assume that it is the function, rather than the brain or body, that makes an experience the type it is. In input-output functionalism, two instances of experience are tokens (exemplars) of the same type of emotion (e.g., anger) because they were evoked by the same stimuli (situations with certain kinds of meaning), because they were caused by the same sorts of cognitive mechanisms (typically called "appraisals"), or because of their relation to behavior (or tendencies to behave). A second form of functionalism offers a more distal or teleological approach to understanding why emotion evolved and what specific and adaptive functional roles it serves in the lives of the individuals and/or species, so that two experiences are tokens of the same emotion because they perform the same function in the life of the person feeling them. By characterizing specific contents of what is experienced (e.g., in anger, people experience their goals as being blocked by another person or by the structure of the situation) in terms of the cognitive processes that produce the content (e.g., in anger, people engage a literal cognitive mechanism for deciding whether or not their goals are blocked), these models are (perhaps unintentionally) reductionistic. Moreover, they stand in contrast to other appraisal models that define appraisals as dimensions of situated meaning that constitute an experience of emotion, rather than cause it.

${ }^{3}$ Scientists sometimes make the mistake of assuming that a scientific description of emotion experience must represent what an emotion feels like with perfect fidelity. No description, however precise, can fully account for the experience of emotion, or for the experience of any conscious content, for that matter. Just as no description will ever allow a color-blind person to experience a color (no matter how much they read descriptions of the content), no scientific description of color instantiates the experience of color. Yet it is possible to study color perception and color experience from a scientific point of view.
} 
different levels of analysis would be to make what Ryle (2000) called a category error. Experiences of emotion can be entirely explained in terms of the neurobiological (material) features of the brain (i.e., they can be causally reduced to brain activity), but they cannot be exclusively equated with any single element or feature such as neural circuitry, biochemical properties of synaptic changes, and so on (i.e., they cannot be ontologically reduced to any material cause). Any conscious event has both neurobiological and phenomenological features. Therefore, knowing about brain activity (or, at another level of analysis, mental processes) alone will not provide a full scientific account of emotion experience. The job of science is to work out the "bridging laws" that link different levels of analysis (Nagel 1961).

Third, conscious states exist only from a first-person point of view. They are ontologically subjective, meaning they only exist when experienced by a conscious agent and cannot be redefined independently of the experiencer. Consequently, they cannot be eliminated in favor of third-person references to instrument-based measures of behavior, physiological activation, or neural events. It is not possible to measure more easily observable aspects of emotion (e.g., facial movements, vocal acoustics, voluntary behaviors, peripheral physiology) to learn something about its subjective aspect. To know what emotion feels like, it is necessary to ask people what they experience. The role of science is to study these subjective events in an epistemologically objective manner.

From the vantage point of biological naturalism, then, scientists must ask questions about subjectively experienced content to understand emotion experience; questions about the material underpinnings of experience will never reveal the entire story. The question "What is the experience of emotion?" is really the question "What do people feel when they feel an emotion?" This is the question of content. What are the correct concepts for capturing or describing the psychological features of the system? Lambie \& Marcel (2002) drew attention to the importance of this question in their recent conceptual analysis of emotion experience. The question "How does the brain instantiate these experiences?" is the straightforward (but difficult) question of how neurophysiological events constitute phenomenological contents. Although science is far from understanding how the brain produces conscious experience, it is possible to sketch the neural circuitry that becomes active during certain types of content. In the next two sections, we address each of these questions in turn. Answers to these two questions are then situated in a psychological framework that describes how experiences of emotion emerge from more basic processes.

\section{THE PHENOMENOLOGICAL CONTENTS OF EMOTION EXPERIENCE}

Not all mental states are conscious, but conscious mental states are mental representations that can, in principle, be reported (Frith et al. 1999), although at any given time they may not be (for a different view, see Lambie \& Marcel 2002). To say that a person is consciously experiencing emotion is to say that he or she has a mental representation of emotion: past feelings (memories), hypothetical feelings (imaginings), or feelings that are occurring in the moment (on-line experiences). The most direct way to measure the contents of a mental representation of emotion is to examine people's verbal behaviors regarding their own mental state, in the form of self-reports (e.g., narratives or simple ratings of emotion words using Likert-type scales). The use of specific words does not directly reveal the contents of the specific mental states to which they refer, but self-reports are a type of communicative act, and communicative acts carry information about a person's internal state that can be inferred by a listener (Wilson \& Sperber 2003), whether the listener is a friend, a therapist, or a scientist. Although self-reports are often not useful to explain why people experience what they do (i.e., self-reports do not reveal causal processes; Nisbett \& Wilson 1977, Wilson \& Dunn 2004), they are useful—and indeed essential—for revealing the ontological structure of consciousness (assuming that you have a willing and able respondent). 
One way that scientists can infer the content in mental states such as experiences of emotion is by treating self-reports as verbal behaviors and examining how people use words to represent those experiences. ${ }^{4}$ Self-report studies, where participants characterize their experiences using emotion words, reveal that states of pleasure or displeasure comprise mental representations of emotion and point to several contents of experience in addition to valence.

\section{Core Affect}

At its core, a mental representation of emotion is a contentful state of pleasure or displeasure (Barrett 2006b,c;Russell 2003;Russell \& Barrett 1999), termed "core affect." The word "core" signifies a form of affective responding that functions as a kind of core knowledge about whether objects or events are helpful or harmful, rewarding or threatening, calling for acceptance or rejection (for a discussion of core knowledge, see Spelke 2000). States of pleasure and displeasure are termed core affect because $(a)$ the capacity to experience pleasure and displeasure is universal to all humans (Mesquita 2003,Russell 1983,Scherer 1997b); ${ }^{5}(b)$ experiences of pleasure and displeasure are present at birth (Emde et al. 1976,Spitz 1965,Sroufe 1979); (c) all instrument-based measures of emotion give evidence of a person's pleasant and unpleasant state or its intensity [e.g., peripheral nervous system activation (Bradley \& Lang 2000;Cacioppo et al. 1997,2000); facial electromyographic activity (Cacioppo et al. 1997, 2000; Messinger 2002); vocal acoustics (Bachorowski 1999); expressive behavior (Cacioppo \& Gardner 1999); and neural activations (Wager et al. 2003); for a review, see (Barrett 2006a)]; (d) pleasure and displeasure constitute a neuropsychologic barometer of the individual's relationship to an environment at a given point in time (Nauta 1971), such that (e) they form the core of consciousness (Edelman \& Tononi 2000; Searle 1992,2004; see A Psychological Framework for Understanding Emotion Experience section below).

\section{The experience of emotion}

affect, perceptions of meaning in the world, and conceptual knowledge about emotion are bound together at a moment in time, producing an intentional state where affect is experienced as having been caused by some object or situation

\section{Core affect}

information about the external world is translated into an internal affective code or state that indicates whether an object or situation is helpful or harmful, rewarding or threatening, requiring approach or withdrawal. With awareness, core affect is experienced as feelings of pleasure or displeasure that are to some extent arousing or quieting. Core affect may be constituted by a constantly changing stream of transient alterations in an organism's

\footnotetext{
${ }^{4}$ Other ways to infer a mental representation of emotion, such as observing a person's face or body, may be adequate at times (cf. Frith et al. 1999,Lambie \& Marcel 2002), but they generally do not capture the full content of experience (Barrett 2006a,Ortony \& Turner 1990,Russell, 2003).

${ }^{5}$ Considerable evidence shows that people can represent their experience in a dialectic fashion (Bagozzi et al. 1999, Kitayama et al. 2000,Schimmack et al. 2002,Scollon et al. 2005), and this has led some to wonder whether pleasure and displeasure really configure as a bipolar dimension of experience. This debate is easily remedied by remembering that a person cannot be aware of two scenes, or objects, or percepts within the same modality at exactly the same moment in time (as illustrated by a Necker cube, Gestalt images such as the young-lady/old-lady ambiguous figure, and incongruent inputs into two eyes in studies of binocular rivalry). So it is with pleasure and displeasure. Conscious experience can move at great speed (estimated at 100-150 ms per conscious moment; Edelman \& Tononi 2000, Gray 2004), so that it is easy to shift back and forth between alternative experiences very quickly, and to summarize both experiences in a memory-based judgment. In fact, research that specifically limits the time window to momentary experience does not find dialectic representations at single moments in time (Leu et al. 2006,Scollon et al. 2005,Yik 2006). As a result, it very unlikely that pleasure and displeasure co-occur in real time, although people can quickly shift experience contents from one moment to the next, and summarize all of the experienced contents in memory. As usual, it all comes down to precision in scientific language, namely, what one means by "at once" in the sentence, "People can (or cannot) feel two things at once." The same argument can be made about emotional complexity, or feeling more than one emotion at once (Charles 2005).
} 
There is mounting empirical evidence that mental representations of emotion have pleasure or displeasure at their core. People are able to give an explicit account of pleasant and unpleasant feelings using a variety of self-rating scales (Barrett \& Russell 1998;Bradley \& Lang 1994;Carroll et al. 1999;Frijda et al. 1989;Kitayama et al. 2000;Lang et al. 1993;Roseman et al. 1996;Russell et al. 1989;Scherer 1997b;Smith \& Ellsworth 1985,1987;Yik et al. 1999). Scales that are explicitly built to measure discrete emotions such as fear, anger, or sadness also provide strong evidence of a common core of pleasant and unpleasant feelings (Barrett \& Russell 1998;Boyle 1986;Feldman 1993,1995a;Mayer \& Gaschke 1988; Russell 1980;Watson \& Clark 1994;Watson \& Tellegen 1985;Zuckerman \& Lubin 1985) (for reviews, see Barrett 2006c,Barrett \& Russell 1999, Russell \& Barrett 1999, Watson et al. 1999). A large experiencesampling project involving idiographic analyses of experiences that were sampled in natural settings over many weeks verified that all participants (approximately 700 American college students) implicitly represented feelings of pleasure and displeasure (Barrett 2006c). This valenced content did not reflect the artificial influence of language (for evidence, see Barrett $2004,2006 b$ ) or social desirability (Barrett 1996), but rather constituted an intrinsic content in mental representations of emotion.

Although core affect is ubiquitous, there are individual and group differences in the degree to which people characterize their experience in terms of pleasure and displeasure. Individuals who are sensitive to the evaluative properties of their surroundings implicitly emphasized the hedonics of their experience (Barrett 2006c). Furthermore, Japanese (compared with American) respondents more often report that they have not experienced any emotional content whatsoever, which suggests that people in these cultures less readily foreground their affective state (Mesquita \& Kawasaka 2002).

\section{From Core Affect to Complex Mental Representations of Emotion}

Core affect is not, in and of itself, sufficient for a mental representation of emotion. An experience of emotion is an intentional state-it is an affective state that is about something. Consequently, any description of emotion experience must go beyond pleasure and displeasure to give a systematic account of the phenomenological differences between emotions that we take to be psychologically distinct, such as anger, sadness, fear, pride, awe, and joy. Studies that focus on emotion words (e.g., angry, sad, afraid, guilty) to describe emotion experiences and studies of appraisals (i.e., the meaning of situations) reveal something about the content of these phenomenological differences. There is still much to be learned about the additional content that constitutes mental representations of emotion, but a brief review of the literature makes clear that mental representations of emotion include representations of arousal as well as relational and situational contents (Fitness \& Fletcher 1993, Mesquita \& Frijda 1992, Shaver et al. 1987,Shweder 1993).

Arousal content-Mental representations of emotion often, but not always, include some arousal-based content (i.e., feeling as if the mind or body is active, as in aroused, attentive, or wound-up, versus feeling that the mind or body is still, as in quiet, still, or sleepy). Felt activation is typically related to, but does not have a one-to-one correspondence with, actual physiologic activity (for a review, see Barrett et al. 2004,Wiens 2005). Cross-sectional studies examining how Western participants self-report their experiences using common English emotion words often give evidence of arousal-based content (for a review, see Russell \& Barrett 1999). It is not yet clear, however, whether arousal is a property of a core affective state (as claimed by Russell 2003, Russell \& Barrett 1999; also see The Neurobiology of Core Affect section below) because idiographic studies of emotion experience indicate that many, but not 
all, participants in Western samples represent feelings of activation and deactivation in experiences of emotion (Barrett 1998,2004;Barrett \& Fossum 2001;Feldman 1995b). In comparison with those who are not very aware of their somatovisceral states, individuals who are interoceptively sensitive emphasize arousal as an aspect of their emotion experience (Barrett et al. 2004) and rate evocative stimuli as more arousing (Pollatos et al. 2005). One possibility is that a person's core affective state can be characterized by arousal (associated with the uncertainty regarding whether a stimulus will predict threat or reward, the need to pay more attention to a stimulus of importance, or an urgency to engage in active coping), but that people vary in their ability or propensity to attend to this property of their core affective state so as to experience it as a feeling.

\section{Arousal content}

an experience of feeling active, aroused, attentive, or wound-up, versus feeling still, as in quiet, still, or sleepy. Does not have a simple one-to-one relationship to objectively measured physical states of arousal

\section{AROUSAL AND THE EXPERIENCE OF EMOTION}

It is often assumed that arousal is essential to the experience of emotion because people perceive emotional feelings in their bodies. William James and later Antonio Damasio proposed that the experience of specific emotions results from the perception of specific and unique patterns of somatovisceral arousal. Schachter and Singer, in contrast, argued that the experience of emotion was due to the direct and explicit experience of a generalized autonomic arousal. Decades of research, however, suggest that neither of these views is correct in the strong sense (for a review, see Barrett et al. 2004). First, little support has been obtained for the idea that different categories of emotion are consistently associated with unique sets of visceral sensations. Second, different measures of autonomic, somatic, or cortical arousal tend not to correlate highly with one another, such that "arousal" is not a unitary phenomenon, suggesting that there is no single accepted definition of arousal. Third, people do not have automatic, immediate, and explicit access to autonomic and somatic activity. As a result, the exact role of bodily feelings in the experience of emotion is still an open scientific question.

Relational content-In self-report studies using emotion words, people report experiencing content related to dominance or submission (Russell \& Mehrabian 1977). A number of studies in which American and Japanese samples rated their experience on English and Japanese words (and their translations) have yielded a dimension of social engagement versus disengagement (e.g., Kitayama et al. 2000,Markus \& Kitayama 1991). Although dominance and social engagement are not synonymous, both are consistent with the relational models in a particular cultural context: Dominance would be the high end of autonomy that is valued in North American cultures, and social engagement would be the high end of harmony or symbiosis that is normative in Japanese cultural contexts (Rothbaum et al. 2000).

Situational content-Mental representations of emotion are intentional states in that they contain some experience of a psychological situation that is perceived by the person to be causally linked to core affective feelings. Appraisals, when they are treated as descriptions (rather than causes) of situated meaning (e.g., Clore \& Ortony 2000,Frijda 2006,Smith \& Ellsworth 1985), provide the best available evidence for mapping the experience of a situation that occurs in a mental representation of emotion. Situational events are experienced as $(a)$ novel or unexpected, $(b)$ conducive or obstructive to some goal, and (c) compatible (or not) 
with norms and values $(d)$ for which a person has (or does not have) some responsibility or agency. A situation is also experienced as calling for some maintenance or change in the behavioral stance (action readiness) where the parameters for actual action are probabilistically certain to some degree. These abstract dimensions of situational meaning show a remarkable degree of convergence across different appraisal models (see Table 29.1 in Ellsworth \& Scherer 2003) and, together with core affect, account for a little less than half of the variance that differentiates categories of emotion experience (e.g., Frijda et al. 1989;Roseman 1991;Roseman et al. 1990,1996;Scherer 1997b;Smith \& Ellsworth 1985,1987) (for reviews, see Mesquita \& Ellsworth 2001,Scherer 1997a). These dimensions of experience also show a remarkable degree of consistency across cultures, although there is variability (e.g., Frijda et al. 1995,Mauro et al. 1992,Mesquita 2001).

\section{Relational content}

the content in an emotion experience that represents the emoter's relationship to another person. In many cases, a mental representation of emotion incorporates the proximity or status of an individual with respect to other individuals present or imagined. Cultural models of relating tend to influence relational content

\section{Situational content}

the meaning of a situation, particularly as it relates to the perceived cause of core affect. Situational content has been mapped using several appraisal dimensions but likely goes beyond these dimensions to reference cultural meanings and practices

Beyond appraisal dimensions-Despite their descriptive value for understanding mental representations of emotion, appraisal dimensions alone do not provide a sufficient account of what people feel when they experience an emotion. First, instances of experience that are categorized as the same emotion, such as anger, are constituted by a variety of experiences of both the physical surroundings and the sociocultural context (Mesquita \& Leu 2006). An experience of anger might indeed involve an insult, where the situation is experienced as obstructing a person's goals, incompatible with his or her identity, and so on, but these abstract descriptions aggregate important phenomenological details that distinguish one feeling of anger from another. You might feel insulted when your friend violates a deeply held belief and you calmly explain your views; when someone cuts you off on the highway and you speed up, yell, or shake your fist; or when someone calls your intelligence into question, causing you to withdraw and quickly leave. The goal for the science of experience is to discover a parsimonious way to describe these variations in anger feelings, but in a fashion that conserves what is meaningfully different about them.

Second, although it is true that respondents across the world can describe emotion experiences in terms of the appraisal dimensions proposed by Western researchers, it is not clear that these dimensions are in fact the most salient aspects of non-Western emotion experiences (Mesquita \& Leu 2006,Shweder \& Haidt 2000). Contentful states of emotion will involve the cultural meanings and practices of self and relating (Markus \& Kitayama 1994,Shweder \& Haidt 2000). In an attempt to capture some of the sociocultural content of experience, a number of studies have added appraisal dimensions that reflect more salient meanings in non-Western cultural contexts (Mesquita \& Ellsworth 2001). For example, esteem by others (status, honor) was an important experience for Surinamese and Turkish immigrants in the Netherlands, but not for Dutch indigenous people (Mesquita 2001). 
Third, the appraisal approach to mapping mental contents is currently limited by the paucity of evidence that appraisal dimensions are adequate representations of emotion as they are experienced in the moment (Parkinson 1997; but see Mesquita \& Kawaska 2002). The overwhelming majority of studies using an appraisal approach ask people to remember or imagine emotion experiences, and self-reports in this context tend to engage semantic knowledge or beliefs about emotion that have an uncertain relationship to the content of emotion experience in real time (Barrett 1997, Robinson \& Clore 2002). As a result, these studies are best understood as measuring the contents that describe a prototypic experience of anger, sadness, fear, and the like, rather than on-line instances of emotion experience per se (for a discussion, see Barrett 2006b).

\section{THE NEURAL REFERENCE SPACE FOR THE EXPERIENCE OF EMOTION}

Ontologically, experiences of emotion are pleasant or unpleasant states that contain additional experiential contents, such as felt arousal, and relational or situational meaning. At present, it is not possible to causally reduce these experiences to neurobiological processes and explain how neural activity instantiates specific emotional contents (or any conscious contents for that matter). It is possible, however, to offer a preliminary sketch of the brain areas that are active during experiences of emotion (i.e., a neural reference space for mental representations of emotion), and we do so in Figure 1. This neural reference space is derived from neuroanatomical studies of the human brain and from neuroimaging studies of emotion experience, including an ongoing meta-analytic effort conducted by Barrett, Wager, and their students. A summary of studies that have imaged the experience of emotion (anger, sadness, fear, disgust, and happiness) and core affective feelings (pleasant and unpleasant affects) in normal participants using a number of different induction techniques (visual, auditory, olfactory, imagery, and memory) has produced a summary activation map (Figure 2) that is similar to the neural reference space for mental representations of emotion hypothesized in Figure $1 .^{6}$ Many of the brain areas depicted in this neural reference space are part of the larger circuitry that entails consciousness more generally, because experiences of emotion are just one type of conscious content.

\section{Neural reference space}

brain areas that show increased activity during a certain type of mental event, and presumably instantiate that mental content

As we discuss in this section, the reference space includes circuitry in a ventral system at the front of the brain that is broadly related (although likely not specific) to core affective feelings of pleasure and displeasure. It is currently not possible to specify the brain areas that correspond to the other conscious contents found in mental representations of anger, sadness, fear, and so on (i.e., arousal-, relational-, and situation-based content). ${ }^{7}$

However, research suggests that mental representations of emotion routinely involve amore dorsal medial prefrontal system that may support the cognitive processes involved with

\footnotetext{
${ }^{6}$ The question of how the brain gives rise to emotion (and the experience of it) is often understood and answered (e.g., LeDoux 1996,Panksepp 1998) as a quest for "essential nodes" or brain systems dedicated to generating specific emotions, such as anger, sadness, fear, and so on. However, research guided by this quest has thus far produced little support for consistency and specificity in the circuitry that supports the experience and perception of emotion in humans, as evidenced by two recent meta-analyses (Murphy et al. 2003,Phan et al. 2002) summarizing the first decade of neuroimaging (fMRI and PET) research on emotion (for a review, see Barrett \& Wager 2006). Although it may be premature to reject the idea of essential nodes for discrete emotions in the brain, it is not prudent to accept that idea too quickly, either.

${ }^{7}$ It is difficult to assess the brain structures associated with arousal-related content because studies typically tend to confound three different types of arousal (i.e., the intensity of a stimulus, feelings of bodily activity, and feelings of alertness).
} 
generating at least some of these contents, specifically conceptualizations of situational cause. Similar ventral and dorsal systems have been discussed previously as the circuitry supporting the generation and regulation of an emotional reaction (Ochsner \& Gross 2005, Yamasaki et al. 2002) or affective state (Phillips et al. 2003).

\section{The Neurobiology of Core Affect}

Figure 2 indicates that mental representations of emotion are consistently associated with increased activation involving a broad swath of the temporal lobe (including the amygdala), orbitofrontal cortex (OFC), and the ventromedial prefrontal cortex (VMPFC) (for definitions, see Figure 1). Together, these areas form a distributed, functional circuit in the ventral portion of the human brain that is involved in establishing the threat or reward value of a stimulus. Value is established by linking sensory information about the stimulus with a representation of how the stimulus affects the person's somatovisceral state (Barbas et al. 2003, Ghashghaei \& Barbas 2002,Kringelbach \& Rolls 2004,Ongur et al. 2003, Ongur \& Price 2000).

Neuroanatomical studies of both primates and humans, along with neuroimaging and lesion evidence in humans, suggest that this ventral system creates a context-sensitive neural representation of an object's value by influencing a person's core affective state to resemble that which has resulted from prior experiences with the object. This view has elements in common with the somatic marker hypothesis (e.g., Bechara et al. 2000) and is consistent with the evidence that OFC plays a role in reinforcement and reversal learning (Kringelbach 2005,Kringelbach \& Rolls 2004).

Although the details remainz to be specified, the available evidence suggests that neural representations of sensory information about a stimulus and its somatovisceral impact are entailed by two related functional circuits that make up the ventral system for core affect (for reviews, see Carmichael \& Price 1996,Elliott et al. 2000, Ongur \& Price 2000). The first functional circuit involves connections between the basolateral (BL) complex of the amygdala, which indelibly codes the original value of a stimulus (Bouton 2005), the central and lateral aspects of the OFC, which is necessary to a flexible, experience-or context-dependent representation of an object's value (Elliott et al. 2000,Kringelbach 2005,Kringelbach \& Rolls 2004,Morris \& Dolan 2004), and the anterior insula, which is involved in representing interoceptive cues (Craig 2002,2003; Dunkley et al. 2005; Wiens 2005). Both the BL and lateral OFC have robust connections with cortical representations of every sensory modality and have strong reciprocal connections (Ghashghaei \& Barbas 2002,Kringelbach \& Rolls 2004,McDonald 1998,Stefanacci \& Amaral 2002), so that they form a functional circuit that integrates sensory (including somatovisceral) information. This information is needed to establish (at least initially) a value-based representation of an object that includes both external sensory features of the object along with its impact on the homeostatic state of the body. One recent formulation argues that the BL complex formulates the predictive value of a stimulus, whereas the OFC participates in generating a response based on that prediction (Holland \& Gallagher 2004).

The second circuit, entailing a neural representation that guides visceromotor control, involves reciprocal connections between the ventromedial prefrontal cortex (VMPFC), including the closely related subgenual anterior cingulate cortex (ACC) and the amygdala, which together modulate the visceromotor (i.e., autonomic, chemical, and behavioral) responses that are part of the value-based representations of an object (Koski \& Paus 2000). VMPFC, in particular, may help to link sensory representations of stimuli and their associated visceromotor (i.e., core affective) outcomes and provides an "affective working memory" whose contents inform choices and judgments contingent upon an assessment of affective value (as computed by the $\mathrm{BL}$ and lateral OFC). This conclusion fits with the finding that VMPFC (particularly the medial sector of the OFC) is important for altering simple stimulus-reinforcer associations via 
extinction (Milad et al. 2005,Phelps et al. 2004,Quirk et al. 2000) or reversal learning (Fellows \& Farah 2003) and is preferentially activated by somatovisceral or interoceptive information (Hurliman \& Parlo 2005) more generally. The representations encoded in VMPFC may also be useful for decisions based on intuitions and feelings rather than on explicit rules (Goel \& Dolan 2003, Shamay-Tsoory et al. 2005), including guesses and familiarity-based discriminations (Elliott et al. 1999,2000; Schnider et al. 2000; Schnyer et al. 2005).

\section{OFC}

$$
\text { orbitofrontal cortex }
$$

VMPFC

ventromedial prefrontal cortex

ACC

anterior cingulate cortex

By virtue of a series of cascading routes, this ventral circuitry projects directly and indirectly (via ventral striatum) to hypothalamus and brainstem areas involved in computing value quickly and efficiently to influence the autonomic, chemical, and behavioral responses that help to establish an affective representation of an object. ${ }^{8}$ The resulting perturbations of the organism's somatovisceral state (or internal milieu) translate information about the external world into an internal affective code or representation (Damasio 1994,1999;Nauta 1971). These representations make up a person's core affective reaction to an object or stimulus, directing the body to prepare for some behavioral response toward that object. A neural representation that entails core affect not only musters attention toward an object (via the brainstem and basal forebrain) (Mesulam 2000,Parvizi \& Damasio 2001), but also enhances visual processing of the object (Amaral et al. 2003,Freese \& Amaral 2005; for a recent review, see Phelps 2006). This core affective state is also available to be experienced and can contribute directly to the contents of conscious experience. Furthermore, the circuitry is not only responsible for entailing a pleasant or unpleasant feeling, but it also may be involved in feelings of arousal, as it controls the degree of cortical arousal associated with feelings of alertness as well as the degree of physiologic arousal that is, at times, associated with feelings of activity and energy.

Although neural representations of core affect provide the substrates for experience, the contents of conscious experience may not directly reflect the operation of any single neural component involved in core affect computations. Consider, for example, that individuals with amygdala lesions do not report alterations in the experience of emotion (Anderson \& Phelps 2002). This might mean that amygdala activity influences experience indirectly by influencing the perception of and memory for emotional events, rather than directly modulating experience per se (Anderson \& Phelps 2001). Alternatively, the amygdala's impact on experience may have been missed by the memory-based measures of emotion used in this study, because such measures rely on semantic knowledge (Barrett 1997, Robinson \& Clore 2002) that is preserved in those with amygdala lesions (Anderson \& Phelps 2000), unless lesions take place during early development (Adolphs et al. 1997). However, many emotion induction experiments do not show increased amygdala activation, and those that do show it find that the increase occurs only early in the emotion induction process, when the stimulus is still being viewed, which

\footnotetext{
${ }^{8}$ The VMPFC, OFC, and both the BL complex and central nucleus of the amygdala project to the ventral striatum (particularly the nucleus accumbens or NAcc shell), which is involved in orchestrating motor control (Grillner et al. 2005) and effortful behavior (Salamone \& Correa 2002;Salamone et al. 2006). There is considerable debate about whether the NAcc dopamine system specifically supports rewarding outcomes, with some arguing that dopamine specifically supports reward behaviors (e.g., Schultz 2004,Schultz et al. 2000) or pleasant, high-arousal affective states (Knutson \& Bhanjim 2006), and with others arguing against the dopamine-reward hypothesis (see Salamone et al., 2005).
} 
suggests that the amygdala activity may not produce core affective experience but rather may be a neural precondition that allows for it.

Furthermore, there are mixed findings regarding the emotion experience of patients with OFC lesions, who report experiencing guilt, shame, and pride, although they do tend to report experiences of embarrassment that are dysregulated from the social context (Beer 2006). Other studies, however, indicate that individuals with OFC lesions report decreased affective reactions involved in empathy (Shamay-Tsoory et al. 2004), decreased regret (Camille et al. 2004), and alterations in experiences of anger and happiness (Berlin et al. 2004); individuals with bilateral OFC lesions report large alterations in emotion experience (Hornak et al. 2003).

\section{The Neurobiology of Emotional Contents Beyond Core Affect}

Today, we know little about the contents that make up experiences of emotion beyond the abstract properties described by appraisal dimensions, and we know even less about the specific neural referents for these experienced contents. We do know, however, that humans are equipped both by nature and by culture with the mechanisms for making mental state attributions of the sort likely to be necessary for producing such contents.

Presumably, during a mental representation of emotion, a person makes an attribution about his or her own core affective state. Recent theoretical treatments have argued that mental representations of emotion emerge when core affective feelings are bound to conceptions of the situation and, in so doing, allow an attribution about the cause of one's core affective state (cf. Barrett 2006b,Frijda 2006,Russell 2003). The word "attribution" usually implies that affect has some priority as being real, while perceived causes about affect are not real, or that affect comes first, with attributions of cause coming later, perhaps via a set of rules that people use in an intentional fashion to figure out why they feel some affective state (Schachter \& Singer 1962). A mental representation of emotion, however, is better thought of as a state of mind that instantaneously emerges when core affective feelings are experienced as causally linked to the psychological situation as it is perceived by the person. This view is consistent with recent evidence from social psychology indicating that perceptions of behavior do not occur independently from inferences about cause, but rather both processes proceed in parallel (Kunda \& Thagard 1996,Lieberman et al. 2002,Reed et al. 1997,Smith \& DeCoster 2000) and likely constrain one another, rendering perceptions of physical actions instantly into psychologically meaningful acts.

As illustrated in Figures 1 and 2, experiences of emotion activate two cortical regions that play a role in mental state attributions: medial prefrontal cortex (MPFC), including both the dorsomedial prefrontal cortex (DMPFC) aspect and the more dorsal extension of the VMPFC aspect, and the ACC. The consistent activation of these areas during experiments that involve the experience of emotion (but not necessarily its report) support the hypothesis that mental state attributions are involved in establishing a mental representation of emotion.

\section{DMPFC}

dorsomedial prefrontal cortex

The functional contributions of the MPFC have yet to be precisely determined, but recent research and theorizing suggest that these brain areas jointly contribute to making mental state attributions (for reviews, see Adolphs 2001,Allman et al. 2001,Blakemore et al. 2004, Gallagher \& Frith 2003,Lane \& Garfield 2005,Lane \& McRae 2004, Ochsner \& Gross 2006), such as when a person makes judgments about or infers the psychological (including emotional) states 
of another person, or monitors, introspects, or makes inferences about his or her own momentto-moment feelings (for a summary of studies, see Ochsner et al. 2004; also see Goldin et al. 2005; Mitchell et al. 2005b,c;Ochsner et al. 2005). Similar activations are observed when individuals process the affective connotations of words (Beauregard et al. 1997; Cato et al. 2004; Crosson et al. 1999,2002), or pictures (for review, see Ochsner et al. 2004), or simulate the mental states (e.g., Mitchell et al. 2005a) and empathize with others (Shamay-Tsoory et al. 2003), presumably because these skills require mental state attributions. Several other lesion studies further suggest that damage to these areas changes the experience of both positive and negative affective feelings (e.g., Bechara et al. 1994,1996; but see Camille et al. 2004,Dunn et al. 2006,Hornak et al. 1996), as well as the experience of emotion (e.g., Hornak et al.

2003, Weniger \& Irle 2002). However, the measurement methods adopted in those studies do not allow for strong conclusions regarding the specific roles that various brain structures play in the experience of emotion.

Figure 2 indicates that both the rostral (so-called affective) and dorsal (or cognitive) ACC (Bush et al. 2000) showed a consistent increase in activation associated with mental representations of emotion. The specific functions of the ACC remain a matter of debate (see Allman 2001,Bush et al. 2000), so that its role in the experience of emotion remains speculative, but the ACC may signal the need to represent mental contents in consciousness so as to reduce conflict or seek greater understanding or control over them (Davidson et al. 2002,Lane \& McRae 2004).

In addition to the MPFC and the ACC, a third cortical region identified as active in mental representations of emotion is the left inferior frontal cortex, also called ventrolateral prefrontal cortex (VLPFC). Several studies of response inhibition, response selection, and working memory have shown that the VLPFC is activated in the context of retrieving, maintaining, monitoring, and manipulating conceptual knowledge stored elsewhere in the brain (Gabrieli et al. 1998,Martin \& Chao 2001,Poldrack et al. 1999, Wagner et al. 2001). VLPFC may play a supporting role to the MPFC, coming into play to retrieve conceptual knowledge about emotion, particularly when selecting an appropriate label for a core affective feeling. This conclusion is consistent with the fact that experiences of emotion consistently produce increased activation in the inferior aspects of BA 45, thought to be important for resolving competition between retrieved representations that are goal-relevant and -irrelevant competitors (Badre et al. 2005).

\section{VLPFC}

ventrolateral prefrontal cortex

Finally, Figure 2 also indicates a fourth cortical region corresponding to posterior cingulate or retrosplenial cortex (BA 31) was active during mental representations of emotion. Although the function of this area remains unclear (Maddock 1999), it may play a role in episodic memory processes supporting the experience of emotion (see, e.g., Mantani et al. 2005). The adjacent precuneus area is associated with self-related mental representations (Cavanna \& Trimble 2006).

\section{A PSYCHOLOGICAL FRAMEWORK FOR UNDERSTANDING EMOTION EXPERIENCE}

The evidence from both content-based and neurobiological (i.e., neuroanatomical, neuroimaging, and neuropsychological) analyses of emotion experience indicates that a mental representation of emotion can be (at least minimally) described as pleasure or displeasure 
experienced in conjunction with other mental contents deriving from mental state attributions (e.g., experiencing the psychological situation in a way that is causally linked to affective feeling). These findings are largely consistent with recent psychological treatments of emotion experience that hypothesize the psychological processes by which mental representations of emotion emerge. The central idea is that a mental representation of emotion on a particular occasion is a continuously changing stream of consciousness in which core affect continuously evolves, interacts with, and mutually constrains construals of the psychological situation (see Barrett 2006b,Barrett et al. 2006,Frijda 2006,Russell, 2003).

Continuously through time, the brain is processing and integrating sensory information from the world, somatovisceral information from the body, and prior knowledge about objects and situations to produce an affective state that is bound to a particular situational meaning, as well as a disposition to act in a particular way. As a result, core affective feelings and construals of the psychological situation very likely are perceptually categorized and experienced as a single unified percept, much like color, depth, and shape are experienced together in object perception. Building on this percept, a mental representation of emotion may be an example of what Edelman (1989) calls "the remembered present."

Across this continuously varying landscape, patterns appear that occasionally constitute the conditions for the experience of emotion. In the view described here, an emotion experience is a conceptual structure stored in memory whose conditions include current perceptions, cognitions, actions, and core affect. A specific emotion conceptualization (e.g., a contextspecific conceptualization of anger) is generated via a top-down, multimodel simulation that reinstates how these conditions have been experienced in the past, and this conceptual representation interacts with the existing affect-situation percept to produce the emergence of an emotion experience. In this way, a situated conceptualization (Barsalou 1999,2003;Barsalou et al. 2003) of emotion (i.e., category knowledge about emotion that is situated in knowledge about the social world and is designed for action; Barsalou et al. 2003,Niedenthal et al., 2005 ) constrains the emerging perceptual categorization (for a discussion, see Barrett 2006b). In the resulting representation, core affect is foregrounded and bound to conceptions of the situation, and in so doing, transforms affect into an intentional state by allowing an attribution about its cause. The resulting experience is an emergent gestalt that corresponds to the colloquial notion of having an emotion.

\section{The remembered present}

Edelman's view of consciousness, where memories are dynamically integrated with and constrain ongoing perceptual processing to form a conscious awareness of each moment

\section{Situated conceptualization}

multimodal, situation-specific conceptual representation of a category exemplar that derives from a highly diversified and flexible conceptual system instantiated by sensory and motor activity

As a result, when you sit around a table with others and a colleague argues against a proposal that you have just offered, and you experience an unpleasant feeling with blood pounding in your ears, it is possible to say what the percept is (e.g., an instance of anger), to make reasonable inferences about it (e.g., you are angry because someone interfered with your goal), to predict how best to act on it (e.g., you are in a business meeting so you keep your voice measured and you do not scowl), and to communicate the experience of it to others (e.g., "I was so angry at today's meeting"). It is the content of these acts of categorization that make one feeling of anger distinct from another feeling of anger and different from any feeling of fear. 
The general idea, then, is that people have affective information about their current relationship to the world, either at a sensory level via homeostatic feedback from the body or via neural representations of prior instances when an object or event predicted some homeo-static change. The affective information is not delivered as a specific interoceptive readout of autonomic activity or anything so precise. Rather, it is a core affective state that gives rise to feelings of pleasure or displeasure (and perhaps activation) that are linked to (but not completely derived from) ongoing automatic evaluations or primary appraisals of the world. The way that people conceptualize their affective state will depend on the knowledge about emotion that they bring to bear when categorizing it. A person might experience his or her core affective state as a particular sort of sadness, anger, or nervousness, depending on the conceptual knowledge that he or she brings to bear in that situation.

This view-that an experience of emotion is a state of mind whose content is at once affective (pleasant or unpleasant) and conceptual (a representation of your relation to the world around you - - is consistent with recent theoretical insights in the neurobiology of consciousness. There is a growing consensus that a conscious experience emerges when a selection of neuronal groups, coding for specific perceptual properties, fire together to form a temporary coalition or assembly of synchronous neural activity (Crick \& Koch 2004,Dehaene \& Changeux 2004,Edelman \& Tononi 2000,Engel \& Singer 2001,Llinas et al. 1998). Reverberating, globally coordinated ("reentrant") neural activity of sufficient intensity and duration allows different sensorial features such as color, shape, sound, smell, and interoceptive cues, and, as we now suggest, core affect, as well as other cognitive contents like beliefs or memories, to bind together into a single experience (but for a dissenting view, see Dennett 1991,Zeki 2003). ${ }^{9}$ When people perceive some object or situation, they have a mental representation of something in the outside world. When core affect is simultaneously foregrounded (for any number of reasons), pleasure or displeasure and perceptions of the world are bound in a meaningful way, yielding a mental representation of emotion. This mental event stands for a person's inference about how psychologically meaningful events in the world are causally linked to his or her core affective feeling. Thus, we suggest that coordinated re-entrant neural activity of sufficient intensity and duration produces a unified conscious content, one type of which is the experience of an emotion.

\section{IMPLICATIONS FOR A SCIENTIFIC AGENDA}

Using the framework provided by Searle's $(1992,2000,2004)$ biological naturalism, we have argued that a scientific understanding of emotion experience requires rich, context-sensitive descriptions of what is experienced, the causal explanations of how those contents are implemented in the human brain, and an explanatory framework that neither reduces one to the other, nor confounds the two. In the following section, we briefly touch on six important implications of this framework for the scientific study of emotion experience.

\section{A Focus on the Heterogeneity of Emotional Life}

By viewing experiences of emotion as conceptual acts, researchers can better map the richness and diversity in mental representations of emotion. Variation in conceptualizing an instance of core affect, whether because of language use, context, culture, or individual differences in prior experience, will produce variation in whether emotions are experienced, which emotions are experienced, and how they are experienced. As a result, it will be important to describe what is constant and what varies in the conceptual system for emotion from one person to the next. We presently know very little about the conceptual system that supports the mental

\footnotetext{
${ }^{9}$ Disunity can occur in rare cases of disorder where there are problems with feature binding, such as Balint's syndrome, in patients with commissurotomy, or in some cases of schizophrenia.
} 
representation of emotion, and we know even less about the neural referents for the conceptual system for emotion.

\section{The Functionality of Perceiving Core Affective Feelings as Emotions}

An individual's momentary conceptualization of core affect, like all categorization, serves some function (even if maladaptive in a given instant). Categorization shapes core affect into a meaningful experience, allowing inferences about what caused the state, how to deal with the situation, and how to communicate efficiently their experiences of core affect to others. A better understanding of this categorization process will yield important insights into the functional nature of emotion experiences.

There are individual differences in the extent to which people represent emotional content over and above feelings of pleasure and displeasure. Studies have documented that people differ in emotional granularity, or the extent to which they characterize their experiences in discrete emotional versus broadly affective terms (Barrett 1998,2004;Barrett et al. 2000,2001;Lane \& Schwartz 1987; Lane et al. 1997). These differences cannot be fully accounted for by verbal intelligence or how well people understand the meaning of emotion words. Individuals high in granularity represent mental contents in addition to valence, whereas those low in granularity represent their experiences primarily as feelings of pleasure or displeasure. Low granularity means that different negative (or different positive) emotion words are used interchangeably to describe the same experience (such that the use of multiple words is not necessarily a sign of the complexity of feelings). Conscious states have high informational value when they can be easily distinguished or differentiated from other different states (Edelman \& Tononi 2000), so that a granular representation of emotion will allow a person to be more functionally effective, in part because it will differentiate among a large repertoire of possible causes for the experienced state, which potentially reduces uncertainty and provides information about what to do next (Barrett \& Gross 2001).

\section{Core Affect is an Intrinsic Aspect of Consciousness}

Those who write about consciousness assume that affect is intrinsic to conscious experience (cf. Edelman \& Tononi 2000; Searle 1992,2004). Pleasure and displeasure are not only mental contents that can be consciously experienced, but also are regulatory factors that play a broader role in selecting the contents of consciousness. Neuroanatomical evidence indicates that the circuitry underlying core affect (see Figure 1) entrains sensory processing by virtue of strong reciprocal projections to the brainstem and basal forebrain systems; these areas, in turn, have diffuse, unidirectional afferent projections to the rest of cortex and can influence the probability that neurons will fire throughout the entire cortical mantle (Mesulam 2000,Parvizi \& Damasio 2001). In this way, core affect can enhance local sensory processing that is stimulus specific, so that a person can effectively and efficiently assess the relevance or value of the stimulus. Thus, areas involved with establishing a core affective state entrain ongoing processing throughout the rest of the cortex, selecting for neuronal assemblies that maximize reward or minimize threat, thereby influencing which contents are experienced in the moment and which are more likely to be stored in long-term memory (Edelman \& Tononi 2000).

\section{Attention and the Experience of Emotion}

Experiences of emotion are not sequenced, discriminable, conscious events distinct from nonemotion experiences. Neurobiological models of consciousness imply that incoming sensory information (such as that which entails a core affective state) modulates a preexisting conscious field rather than generating it anew (Llinas et al. 1998). This stream of core affect can be a central or a background feature (figure or ground) of consciousness, depending on where attention is applied. When core affect is in the background, it functions as background feelings (Lane \& Garfield 2005) or background emotions (Damasio 1999) that color conscious 
experience in a less direct fashion but presumably have the potential to influence behavior implicitly (Berridge \& Winkielman 2003, Winkielman et al. 2005). Backgrounded core affect is experienced as a property of the external world rather than as the person's reaction to it. We experience some people as nice and others as mean, some foods as delicious but others as distasteful, some pictures as pleasing and others as negative. It may be under these circumstances that core affect directly translates into a behavioral response. When core affect is foregrounded, it can be experienced directly as pleasant or unpleasant content and can serve as information for making explicit judgments and decisions (Clore et al. 2005,Schwarz \& Clore 1983), or core affective feelings can be attributed to some situational cause, thereby forming the basis of an emotion experience (cf. Barrett 2006b,Russell 2003; but see Frijda 2005,Lambie $\&$ Marcel 2002, who characterize backgrounded affect as emotion experience). Core affective feelings will be foregrounded, either because of their intensity, because of a goal to introspect, or because the situation cues attention. Clearly, a key question for future research is when and how foregrounding happens to produce the experience of "having an emotion."

\section{Common Substrates for Psychopathology}

It seems likely at this point that many psychological disorders share a common or "transdiagnostic" (Harvey et al. 2004) disturbance in core affective processing (Barlow 2002) that involves vigilance to threat (Harvey et al. 2004,Quigley \& Barrett 1999), is on a continuum with normal personality variability (Weinstock \& Whisman 2006), and is linked to the short allele of a serotonin transporter (5-HTT) gene which produces an affective vulnerability to environmental stress (for a review, see Hariri \& Holmes 2006). The brain structures and neurotransmitters (such as serotonin and dopamine) associated with the ventral system for core affect are implicated in a range of psychopathologies characterized by affective disturbances, including depression (e.g., Anand et al. 2005, Drevets 2000,Drevets et al. 1997,Hariri et al. 2005,Lacerda et al. 2004,Mayberg 1997,Pezawas et al. 2005), schizophrenia (e.g., Fahim et al. 2005a,b), obsessive-compulsive disorder (e.g., Nakao et al. 2005, Valente et al. 2005), posttraumatic stress disorder (e.g., Bryant et al. 2005; Rauch et al. 2000; Shin et al. 2004,2005), social anxiety and generalized anxiety disorder (for a review see Stein et al. 2002), and panic disorder (e.g., Kent et al. 2005). Such a transdisorder affective syndrome, if it exists, might also predispose people to health-related problems as well (Gallo \& Matthews 2003,Pressman \& Cohen 2005).

Furthermore, which emotion is experienced, and how it is experienced, is a matter of intentional focus and interpretation, so that the conceptualization process may also act as a transdisorder vulnerability to mood-related disorders. Conceptualizing core affect might be thought of as a skill, in that some people are better than are others at tailoring conceptual knowledge to meet the needs of socially situated action (Barrett 2006b). This skill for wielding conceptual knowledge about emotion might be considered a core aspect of emotional intelligence (Barrett $\&$ Gross 2001) and is a central feature of emotion-focused psychotherapeutic treatments (Greenberg 1993,Moses \& Barlow 2006).

\section{MORE ON THE FOREGROUNDING OF CORE AFFECT}

The mechanisms by which affect is foregrounded or backgrounded remain to be specified, but it is generally accepted that an "attentional matrix" (Mesulam 2000) within the brain foregrounds particular contents of consciousness (whether core affect and/or beliefs about core affect) by modulating the intensity of neural firing in coalitions of neurons. This attentional matrix includes not only the core affect-driven bottom-up form of attention (supported by the brainstem and basal forebrain; Edelman \& Tononi 2000,Parvizi \& Damasio 2001), but also a top-down form of attention (supported by dorsolateral prefrontal, anterior cingulate, and parietal cortices) that is entrained by sensory stimulation of sufficient intensity or driven by an individual's processing goals (Crick \& Koch 2004,Dehaene \& 
Changeux 2004,Maia \& Cleeremans 2005,Naghavi \& Nyberg 2005). Whatever the mix of attentional factors, each person has one seamless flow of experience that is continually changing and can be more or less infused with some sort of affective content, depending on the focus of attention.

\section{Cognition and the Experience of Emotion}

Finally, the distinction between cognitive activity and emotion experience is probably better conceptualized as more of a gradient rather than two independent systems that can interact with one another. Although scientists are very used to thinking about cognitive events (such as thoughts, memories, and beliefs) as separate from emotional events, this distinction is probably phenomenological rather than causal and does not seem to be respected by the brain. Brain structures at the heart of the neural circuitry for emotion (e.g., the amygdala) impact cognitive processing from early attention allocation (Holland \& Gallagher 1999) through perceptual processing to memory (for a recent review, see Phelps 2006). Similarly, brain structures involved in the neural circuitry for cognition, such as DMPFC and VLPFC, have an intrinsic role in the experience of emotion (see Figure 2). Decision-making processes that are traditionally thought of in cognitive terms, such as moral reasoning, seem to have core affect as their basis (Greene et al. 2004,Haidt 2001), and unrelated experiences of emotion can color such diverse outcomes as economic decisions (Loewenstein \& Lerner 2003) and stereotyping (e.g., Bodenhausen \& Moreno 2000,DeSteno et al. 2004). Consider the distinction between feeling and thinking, compared with other phenomenological boundaries respected by the brain, such as visual and auditory processing. No one would ever mistake seeing for hearing (although one sensory representation might trigger another), but the same cannot be said for feeling and thinking.

\section{SUMMARY}

In much scientific writing about emotion, everyday words for emotion, such "anger,"

"sadness," and "fear," are used as technical terms to refer to both conscious events and to causal (i.e., behavioral, cognitive, or neurological) events, without a detailed exposition about how the two are related, because one is literally redefined in terms of the other. Although this approach has been expedient, it has left emotion experiences, a fundamental centerpiece in human existence, largely underspecified from a scientific standpoint.

In this chapter, we began by locating the study of emotion experience in a philosophical approach to understanding consciousness, because questions about emotion experience are essentially questions about consciousness and discussions about the nature of emotion experience are always grounded in some philosophical perspective, even if implicitly. Using Searle's $(1992,2000,2004)$ biological naturalism, we argued that building a scientific model of emotion experience requires both a descriptive psychology of mental contents and a detailed neurobiology that entails them. We believe that words refer to mental states but not to the mechanisms that generated those states (Barrett 2006b,Russell 2003,Sabini \& Silver 2005). Thus, we have argued that emotion words are not the names of things - rather they demarcate mental representations that are constituted as feelings of pleasure or displeasure and socially situated conceptualizations of emotion.

We then outlined what is currently known regarding $(a)$ psychological descriptions of the contents of mental representations of emotion and $(b)$ the neural reference space (based on neuroanatomical, neuroimaging, and, to some degree, neuropsychological findings) that is correlated with those contents. In addition, we framed these findings within an emerging psychological model for the experience of emotion that describes mental representations of emotion as emergent phenomena, constructed from more basic affective and conceptual 
representations, and we discussed how the binding of core affect and conceptual knowledge might arise naturally from the neurobiological processes that allow neural activity to give rise to conscious content. Taken together, this framework sets a scientific agenda that affords several advantages for a psychological understanding of emotion experiences as real, potent, and important aspects of conscious life, as well as for understanding the role of emotion experience in the economy of the mind and behavior.

\section{SUMMARY POINTS}

1. A scientific understanding of emotion experience requires a rich, context-sensitive description of what is experienced, a causal explanation of how experienced content is constituted by the human brain, and an explanatory framework that neither reduces one to the other nor confounds the two.

2. At its core, the experience of emotion can be described a contentful state of pleasure or displeasure. Some degree of arousal may also be experienced. Some situation with a specific relational meaning is experienced simultaneously with and as having caused these affective feelings.

3. At present, it is not possible to explain how neural activity instantiates specific emotional contents (or any conscious content, for that matter). It is possible, however, to offer a preliminary sketch of a neural reference space for mental representations of emotion.

4. The available evidence on content and neurobiological accounting of emotion experience can be integrated into a broad framework that describes, in psychological terms, how the experience of emotion emerges from more basic processes. Core affect and construals of the psychological situation are perceptually categorized and experienced as a single unified percept. Conceptual knowledge about emotion constrains perceptual processing to shape the emergence of an experience of emotion.

\section{FUTURE ISSUES}

1. Regarding core affect: Is core affect a natural kind category? Are other contents, such as arousal, fundamental properties of core affect? Is there only one kind of pleasure (or displeasure) or many phenomenologically distinct feelings? What are processes by which and conditions for when affect is backgrounded and experienced as a property of the world or foregrounded and experienced as a property of the self?

2. Regarding other aspects of the psychological content of emotion experience: Beyond the abstract properties described by appraisal dimensions, what is the additional content that constitutes mental representations of emotion? Can any cognitive content play a role in shaping an emotion experience?

3. Regarding the neurobiological bases of emotion: Is the neural reference space misspecified, or incomplete? How do neurobiological processes give rise to the contents of emotion experience?

4. Regarding the mental representation of emotion: When and why is core affect conceptualized as emotion? What is the structure and content of the conceptual system that supports the mental representation of emotion and the neural referents that support this conceptual system? What are the individual differences in the content, structure, and function of the conceptual system? Do they relate to 
observed differences in emotional granularity, and what is their functional consequence? How do affect and conceptual knowledge about emotion constrain one another in real-time processing?

\section{ACKNOWLEDGMENTS}

This work was supported by an NSF grant (BCS 0527440) and an NIMH Independent Scientist Research Award (K02 MH001981) to Lisa Feldman Barrett and NIH grants (R01 MH58147 and R01 MH66957) to James Gross. The authors wish to thank William Irwin for preparing Figure 1, Tor Wager for his permission to report the preliminary metaanalytic findings presented in Figure 2, Larry Barsalou for contributing some of the wording in the description of situated conceptualizations of emotion, as well as Gerald Clore, Chris Wright, Nico Frijda, Tony Marcel, Jeanne Tsai, Seth Duncan, Kristen Lindquist, Eliza Bliss-Moreau, and Jennifer Mize for their comments on earlier drafts of this paper.

\section{LITERATURE CITED}

Adolphs R. The neurobiology of social cognition. Curr. Opin. Neurobiol 2001;11:231-39. [PubMed: 11301245]

Adolphs R, Lee GP, Tranel D, Damasio AR. Bilateral damage to the human amygdala early in life impairs knowledge of emotional arousal. Soc. Neurosci. Abstr 1997;23:1582.

Allman JM, Hakeem A, Erwin JM, Nimchinsky E, Hoff P. The anterior cingulate cortex: the evolution of an interface between emotion and cognition. Ann. NY Acad. Sci 2001;935:107-17. [PubMed: 11411161]

Amaral DG, Behniea H, Kelly JL. Topographical organization of projections from the amygdala to the visual cortex in the Macaque monkey. Neuroscience 2003;118:1099-120. [PubMed: 12732254]

Anand A, Li Y, Wang Y, Wu J, Gao S, et al. Activity and connectivity of brain mood regulating circuit in depression: a functional magnetic resonance study. Biol. Psychiatry 2005;57:1079-88. [PubMed: 15866546]

Anderson AK, Phelps EA. Expression without recognition: contributions of the human amygdala to emotional communication. Psychol. Sci 2000;11:106-11. [PubMed: 11273416]

Anderson AK, Phelps EA. Lesions of the human amygdala impair enhanced perception of emotionally salient events. Nature 2001;411:305-9. [PubMed: 11357132]

Anderson AK, Phelps EA. Is the human amygdala critical for the subjective experience of emotion? Evidence of intact dispositional affect in patients with amygdala lesions. J. Cogn. Neurosci 2002;14:709-20. [PubMed: 12167256]

Arnold, M. Emotion and Personality. Columbia Univ. Press; New York: 1960.

Bachorowski J. Vocal expression and perception of emotion. Curr. Dir. Psychol. Sci 1999;8:53-56.

Badre D, Poldrack RA, Pare-Blagoev EJ, Insler RZ, Wagner AD. Dissociable controlled retrieval and generalized selection mechanisms in ventrolateral prefrontal cortex. Neuron 2005;47:907-18. [PubMed: 16157284]

Bagozzi RP, Wong KS, Youjae Y. The role of culture and gender in the relationship between positive and negative affect. Cogn. Emot 1999;13:641-72.

Barbas H, Saha S, Rempel-Clower N, Ghashghaei T. Serial pathways from primate prefrontal cortex to autonomic areas may influence emotional expression. BMC Neurosci 2003;4:25. [PubMed: 14536022]

Barlow, DH. Anxiety and Its Disorders: The Nature and Treatment of Anxiety and Panic. Guilford; New York: 2002.

Barrett LF. Hedonic tone, perceived arousal, and item desirability: three components of affective experience. Cogn. Emot 1996;10:47-68.

Barrett LF. The relationships among momentary emotion experiences, personality descriptions, and retrospective ratings of emotion. Personal. Soc. Psychol. Bull 1997;23:1100-10.

Barrett LF. Discrete emotions or dimensions? The role of valence focus and arousal focus. Cogn. Emot 1998;12:579-99. 
Barrett LF. Feelings or words? Understanding the content in self-report ratings of experienced emotion. J. Personal. Soc. Psychol 2004;87:266-81.

Barrett LF. Emotions as natural kinds? Perspect. Psychol. Sci 2006a;1:28-58.

Barrett LF. Solving the emotion paradox: categorization and the experience of emotion. Personal. Soc. Psychol. Rev 2006b;10:20-46.

Barrett LF. Valence as a basic building block of emotional life. J. Res. Personal 2006c;40:35-55.

Barrett LF, Fossum T. Mental representations of affect knowledge. Cogn. Emot 2001;15:333-63.

Barrett, LF.; Gross, JJ. Emotional intelligence: a process model of emotion representation and regulation. In: Mayne, TJ.; Bonanno, GA., editors. Emotions: Current Issues and Future Directions. Guilford; New York: 2001. p. 286-310.

Barrett LF, Gross J, Christensen TC, Benvenuto M. Knowing what you're feeling and knowing what to do about it: mapping the relation between emotion differentiation and emotion regulation. Cogn. Emot 2001;15:713-24.

Barrett LF, Lane RD, Sechrest L, Schwartz GE. Sex differences in emotional awareness. Personal. Soc. Psychol. Bull 2000;26:1027-35.

Barrett, LF.; Niedenthal, PM.; Winkielman, P., editors. Emotion and Consciousness. Guilford; New York: 2005.

Barrett LF, Quigley K, Bliss-Moreau E, Aronson KR. Arousal focus and interoceptive sensitivity. J. Personal. Soc. Psychol 2004;87:684-97.

Barrett LF, Russell JA. Independence and bipolarity in the structure of current affect. J. Personal. Soc. Psychol 1998;74:967-84.

Barrett LF, Russell JA. The structure of current affect: controversies and emerging consensus. Curr. Dir. Psychol. Sci 1999;8:10-14.

Barrett LF, Wager T. The structure of emotion: evidence from the neuroimaging of emotion. Curr. Dir. Psychol. Sci 2006;15:79-85.

Barsalou LW. Perceptual symbol systems. Behav. Brain Sci 1999;22:577-660. [PubMed: 11301525]

Barsalou LW. Situated simulation in the human conceptual system. Lang. Cogn. Process. Spec. Issue Concept. Represent 2003;18:513-62.

Barsalou, LW.; Niedenthal, PM.; Barbey, A.; Ruppert, J. Social embodiment. In: Ross, B., editor. The Psychology of Learning and Motivation. Academic; San Diego: 2003. p. 43-92.

Beauregard M, Chertkow H, Bub D, Murtha S, Dixon R, Evans A. The neural substrate for concrete, abstract, and emotional word lexica: a positron emission tomography study. J. Cogn. Neurosci 1997;9:441-61.

Bechara A, Damasio AR, Damasio H, Anderson SW. Insensitivity to future consequences following damage to human prefrontal cortex. Cognition 1994;50:7-15. [PubMed: 8039375]

Bechara A, Damasio H, Damasio AR. Emotion, decision making and the orbitofrontal cortex. Cereb. Cortex 2000;10:295-307. [PubMed: 10731224]

Bechara A, Tranel D, Damasio H, Damasio A. Failure to respond autonomically to anticipated future outcomes following damage to prefrontal cortex. Cereb. Cortex 1996;6:215-25. [PubMed: 8670652]

Beer, JS. The importance of emotion-social cognition interactions for social functioning: insights from orbitofrontal cortex. In: Jones, EH.; Winkielman, P., editors. Fundamentals of Social Neuroscience. MIT Press; Cambridge, MA: 2006. In press

Berlin HA, Rolls ET, Kischka U. Impulsivity, time perception, emotion and reinforcement sensitivity in patients with orbitofrontal cortex lesions. Brain 2004;127:1108-26. [PubMed: 14985269]

Berridge KC, Winkielman P. What is an unconscious emotion? (The case for unconscious "liking."). Cogn. Emot 2003;17:181-11.

Blakemore SJ, Winston J, Frith U. Social cognitive neuroscience: Where are we heading? Trends Cogn. Sci 2004;8:216-22. [PubMed: 15120680]

Bodenhausen, GV.; Moreno, KN. How do I feel about them: the role of affective reactions in intergroup perception. In: Bless, H.; Forgas, JP., editors. The Message Within: Subjective Experience in Social Cognition and Behavior. Psychol. Press; Philadelphia, PA: 2000. p. 283-303.

Bouton ME. Behavior systems and the contextual control of anxiety, fear, and panic. 2005:205-27.See Barrett et al. 2005 
Boyle GJ. Higher-order factors in the Differential Emotions Scale (DES-III). Personal. Individ. Differ 1986;7:305-10.

Bradley, MM.; Lang, PJ. Measuring emotion: behavior, feeling, and physiology. In: Lane, RD.; Nadel, L.; Ahern, GL.; Allen, JJB.; Kaszniak, AW.; Rapcsak, SZ.; Schwartz, GE., editors. Cognitive Neuroscience of Emotion. Oxford Univ. Press; New York: 2000. p. 242-76.

Bradley MM, Lang PJ. Measuring emotion: the self-assessment manikin and the semantic differential. J. Behav. Therapy Exper. Psychiatry 1994;22:49-59.

Bryant RA, Felmingham KL, Kemp AH, Barton M, Peduto AS, et al. Neural networks of information processing in posttraumatic stress disorder: a functional magnetic resonance imaging study. Biol. Psychiatry 2005;52:111-18. [PubMed: 16038681]

Buck R. The biological affects: a typology. Psychol. Rev 1999;106:301-36. [PubMed: 10378015]

Bush G, Luu P, Posner MI. Cognitive and emotional influences in anterior cingulate cortex. Trends Cogn. Sci 2000;4:215-22. [PubMed: 10827444]

Cacioppo JT, Berntson GG, Klein DJ, Poehlmann KM. The psychophysiology of emotion across the lifespan. Annu. Rev. Gerontol. Geriatr 1997;17:27-74.

Cacioppo, JT.; Berntson, GG.; Larsen, JT.; Poehlmann, KM.; Ito, TA. The psychophysiology of emotion. In: Lewis, M.; Haviland-Jones, M., editors. The Handbook of Emotion. Guilford; New York: 2000. p. 173-91.

Cacioppo JT, Gardner WL. Emotion. Annu. Rev. Psychol 1999;50:191-214. [PubMed: 10074678]

Camille N, Coricelli G, Sallet J, Pradat-Diehl P, Duhamel JR, Sirigu A. The involvement of the orbitofrontal cortex in the experience of regret. Science 2004;304:1167-70. [PubMed: 15155951]

Carmichael ST, Price JL. Connectional networks within the orbital and medial prefrontal cortex of macaque monkeys. J. Comp. Neurol 1996;371:179-207. [PubMed: 8835726]

Carroll JM, Yik MSM, Russell JA, Barrett LF. On the psychometric principles of affect. Rev. Gen. Psychol 1999;3:14-22.

Cato MA, Crosson B, Gokcay D, Soltysik D, Wierenga C, et al. Processing words with emotional connotation: an FMRI study of time course and laterality in rostral frontal and retrosplenial cortices. J. Cogn. Neurosci 2004;16:167-77. [PubMed: 15068589]

Cavanna AE, Trimble MR. The precuneus: a review of its functional anatomy and behavioral correlates. Brain 2006;129:564-83. [PubMed: 16399806]

Charles ST. Viewing injustice: greater emotional heterogeneity with age. Psychol. Aging 2005;20:159_ 64. [PubMed: 15769221]

Clore, GL.; Ortony, A. Cognition in emotion: always, sometimes, or never?. In: Lane, RD.; Nadel, L., editors. Cognitive Neuroscience of Emotion. London Univ. Press; London: 2000. p. 24-61.

Clore GL, Storbeck J, Robinson MD, Centerbar DB. Seven sins in the study of unconscious affect. 2005:384-408.See Barrett et al. 2005

Craig AD. How do you feel? Interoception: the sense of the physiological condition of the body. Nat. Neurosci 2002;3:655-66.

Craig AD. Interoception: the sense of the physiological condition of the body. Curr. Opin. Neurobiol 2003;13:500-5. [PubMed: 12965300]

Crick, FC.; Koch, C. A framework for consciousness. In: Gazzaniga, MS., editor. The Cognitive Neurosciences. MIT Press; Cambridge, MA: 2004. p. 1133-44.

Crosson B, Cato MA, Sadek JR, Gokcay D, Bauer RM, et al. Semantic monitoring of words with emotional connotation during fMRI: contribution of anterior left frontal cortex. J. Int. Neuropsychol. Soc 2002;8:607-22. [PubMed: 12164671]

Crosson B, Radonovich K, Sadek JR, Gokcay D, Bauer RM, et al. Left-hemisphere processing of emotional connotation during word generation. Neuroreport 1999;10:2449-55. [PubMed: 10574350]

Damasio, AR. Descartes' Error: Emotion, Reason, and the Human Brain. Grossett/Putnam; New York: 1994.

Damasio, AR. The Feeling of What Happens: Body and Emotion in the Making of Consciousness. Harcourt Brace; New York: 1999.

Davidson RJ, Pizzagalli D, Nitschke JB, Putnam K. Depression: perspectives from affective neuroscience. Annu. Rev. Psychol 2002;53:545-74. [PubMed: 11752496] 
DeArmond, SJ.; Fusco, MM.; Dewey, MM. Structure of the human brain: a photographic atlas. 3rd ed.. Oxford Univ. Press; New York: 1989.

Dehaene, S.; Changeux, JP. Neural mechanisms for access to consciousness. In: Gazzaniga, MS., editor. The Cognitive Neurosciences. MIT Press; Cambridge, MA: 2004. p. 1145-58.

Dennett, DC. Consciousness Explained. Little, Brown; Boston: 1991.

DeSteno D, Dasgupta N, Bartlett MY, Cajdric A. Prejudice from thin air: the effect of emotion on automatic intergroup attitudes. Psychol. Sci 2004;15:319-24. [PubMed: 15102141]

Drevets WC. Neuroimaging studies of mood disorders. Biol. Psychiatry 2000;48:813-29. [PubMed: 11063977]

Drevets, WC.; Gadde, K.; Krishman, R. Neuroimaging studies of depression. In: Charney, DS.; Nestler, EJ.; Bunney, BJ., editors. Neurobiology of Mental Illness. Oxford Univ. Press; New York: 1997. p. 461-90.

Dunkley P, Wise RG, Aziz Q, Painter D, Brooks J, et al. Cortical processing of visceral and somatic stimulation: differentiating pain intensity from unpleasantness. Neuroscience 2005;133:533-42. [PubMed: 15896917]

Dunn BD, Dalgleish T, Lawrence AD. The somatic marker hypothesis: a critical evaluation. Neurosci. Biobehav. Rev 2006;30:239-71. [PubMed: 16197997]

Edelman, GM. The Remembered Present. Basic; New York: 1989.

Edelman, GM.; Tononi, G. A Universe of Consciousness. Basic; New York: 2000.

Ekman, P. Universals and cultural differences in facial expressions of emotion. 1971. In: Cole, JR., editor. Nebraska Symposium on Motivation. Univ. Nebraska Press; Lincoln: 1972. p. 207-83.

Ekman P. Are there basic emotions? Psychol. Rev 1992;99:550-53. [PubMed: 1344638]

Elliott R, Dolan RJ, Frith CD. Dissociable functions in the medial and lateral orbitofrontal cortex: evidence from human neuroimaging studies. Cereb. Cortex 2000;10:308-17. [PubMed: 10731225]

Elliott R, Rees G, Dolan RJ. Ventromedial prefrontal cortex mediates guessing. Neuropsychologia 1999;37:403-11. [PubMed: 10215087]

Ellsworth, PC.; Scherer, KR. Appraisal processes in emotion. In: Goldsmith, H.; Davidson, RJ.; Scherer, KR., editors. Handbook of the Affective Sciences. Oxford Univ. Press; New York: 2003. p. 572-95.

Emde RN, Gaensbauer TJ, Harmon RJ. Emotional expression in infancy: a biobehavioral study. Psychol. Issues 1976;10:1-200. [PubMed: 959439]

Engel AK, Singer W. Temporal binding and the neural correlates of sensory awareness. Trends Cogn. Sci 2001;5:16-25. [PubMed: 11164732]

Fahim C, Stip E, Mancini-Marie A, Gendron A, Mensour B, Beauregard M. Differential hemodynamic brain activity in schizophrenia patients with blunted affect during quetiapine treatment. J. Clin. Psychopharmacol 2005a;25:367-71. [PubMed: 16012281]

Fahim C, Stip E, Mancini-Marïe A, Mensour B, Boulay LJ, et al. Brain activity during emotionally negative pictures in schizophrenia with and without flat affect: an fMRI study. Psychiatr. Res. Neuroimag 2005b;140:1-15.

Feldman LA. Distinguishing depression from anxiety in self-report: evidence from confirmatory factor analysis on nonclinical and clinical samples. J. Consult. Clin. Psychol 1993;69:153-66.

Feldman LA. Variations in the circumplex structure of mood. Personal. Soc. Psychol. Bull 1995a;21:80616.

Feldman LA. Valence focus and arousal focus: individual differences in the structure of affective experience. J. Personal. Soc. Psychol 1995b;69:153-66.

Fellows LK, Farah MJ. Ventromedial frontal cortex mediates affective shifting in humans: evidence from a reversal learning paradigm. Brain 2003;126:1830-37. [PubMed: 12821528]

Fitness J, Fletcher GJO. Love, hate, anger, and jealousy in close relationships: a prototype and cognitive appraisal analysis. J. Personal. Soc. Psychol 1993;65:942-58.

Freese JL, Amaral DG. The organization of projections from the amygdala to visual cortical areas TE and V1 in the macaque monkey. J. Comp. Neurol 2005;486:295-317. [PubMed: 15846786]

Frijda, NH. The Emotions. Cambridge Univ. Press; Cambridge: 1986.

Frijda NH. Emotion experience. Cogn. Emot 2005;19:473-97. 
Frijda, NH. The Laws of Emotion. Erlbaum; Mahwah, NJ: 2006.

Frijda NH, Kuipers P, ter Schure E. Relations among emotion, appraisal, and emotional action readiness. J. Personal. Soc. Psychol 1989;57:212-28.

Frijda, NH.; Markam, S.; Sato, K.; Wiers, R. Emotion and emotion words. In: Russell, JA.; FernandezDols, J-M.; Manstead, ASR.; Wellencamp, JC., editors. Everyday Conceptions of Emotion. Kluwer Acad.; Boston: 1995. p. 121-43.

Frith C, Perry R, Lurner E. The neural correlates of conscious experience: an experimental framework. Trends Cogn. Sci 1999;3:105-14. [PubMed: 10322462]

Gabrieli JD, Poldrack RA, Desmond JE. The role of left prefrontal cortex in language and memory. Proc. Natl. Acad. Sci. USA 1998;95:906-13. [PubMed: 9448258]

Gallagher HL, Frith CD. Functional imaging of “theory of mind.”. Trends Cogn. Sci 2003;7:77-83. [PubMed: 12584026]

Gallo LC, Matthews KA. Understanding the association between socioeconomic status and physical health: Do negative emotions play a role? Psychol. Bull 2003;129:10-51. [PubMed: 12555793]

Ghashghaei HT, Barbas H. Pathways for emotion: interactions of prefrontal and anterior temporal pathways in the amygdala of the rhesus monkey. Neuroscience 2002;115:1261-79. [PubMed: 12453496]

Goel V, Dolan RJ. Reciprocal neural response within lateral and ventral medial prefrontal cortex during hot and cold reasoning. Neuroimage 2003;20:2314-21. [PubMed: 14683732]

Goldin PR, Hutcherson CAC, Ochsner KN, Glover GH, Gabrieli JDE, Gross JJ. The neural bases of amusement and sadness: a comparison of block contrast and subject-specific emotion intensity regression approaches. Neuroimage 2005;27:26-39. [PubMed: 15890534]

Gray, JA. Consciousness. Oxford Univ. Press; New York: 2004.

Greenberg, LS. Emotion and change processes in psychotherapy. In: Lewis, M.; Haviland, JM., editors. Handbook of Emotion. Guilford; New York: 1993. p. 499-508.

Greene JD, Nystrom LE, Engell AD, Darley JM, Cohen JD. The neural bases of cognitive conflict and control of moral judgment. Neuron 2004;44:389-400. [PubMed: 15473975]

Grillner S, Hellgren J, Menard A, Saitoh K, Wilstrom MA. Mechanisms for selection of basic motor programs—roles for the striatum and pallidum. Trends Neurosci 2005;28:364-70. [PubMed: 15935487]

Haidt J. The emotional dog and its rational tail: a social intuitionist approach to moral judgment. Psychol. Rev 2001;108:814-34. [PubMed: 11699120]

Hariri AR, Drabant EM, Munoz KE, Kolachana BS, Mattay VS, et al. A susceptibility gene for affective disorders and the response of the human amygdala. Arch. Gen. Psychiatry 2005;62:146-52. [PubMed: 15699291]

Hariri AR, Holmes A. Genetics of emotional regulation: the role of the serotonin transporter in neural function. Trends Cogn. Sci. 2006In press

Harvey, AG.; Watkins, E.; Mansell, W.; Shafran, R. Cognitive Behavioural Processes Across Psychological Disorders: A Transdiagnostic Approach to Research and Treatment. Oxford Univ. Press; New York: 2004.

Holland PC, Gallagher M. Amygdala circuitry in attentional and representational processes. Trends Cogn. Sci 1999;3:65-73. [PubMed: 10234229]

Holland PC, Gallagher M. Amygdala-frontal interactions and reward expectancy. Curr. Opin. Neurobiol 2004;14:148-55. [PubMed: 15082318]

Hornak J, Bramam J, Rolls ET, Morris RG, O'Dohery J, et al. Changes in emotion after circumscribed surgical lesions of the orbitofrontal and cingulate cortices. Brain 2003;126:1691-712. [PubMed: 12805109]

Hornak J, Rolls ET, Wade D. Face and voice expression identification in patients with emotional and behavioural changes following ventral frontal lobe damage. Neuropsychologia 1996;34:247-61. [PubMed: 8657356]

Hurliman E, Nagode JC, Pardo JV. Double dissociation of exteroceptive and interoceptive feedback systems in the orbital and ventromedial prefrontal cortex of humans. J. Neurosci 2005;25:4641-48. [PubMed: 15872112] 
Izard, CE. Human Emotions. Plenum; New York: 1977.

Izard CE. Four systems for emotion activation: cognitive and noncognitive processes. Psychol. Rev 1993;100:68-90. [PubMed: 8426882]

James, W. The Principles of Psychology. Holt; New York: 1890.

Kent JM, Coplan JD, Mawlawi O, Martinez JM, Browne ST, et al. Prediction of panic response to a respiratory stimulant by reduced orbitofrontal cerebral blood flow in panic disorder. Am. J. Psychiatry 2005;162:1379-81. [PubMed: 15994724]

Kitayama S, Markus HR, Kurokawa M. Culture, emotion and well-being: good feelings in Japan and the United States. Cogn. Emot 2000;14:93-124.

Knutson, B.; Bhanji, J. Neural substrates for emotional traits?. In: Canli, T., editor. Biology of Personality Individual Difference. Guilford; New York: 2006. p. 116-32.

Koski L, Paus T. Functional connectivity of the anterior cingulate cortex within the human frontal lobe: a brain-mapping meta-analysis. Exp. Brain Res 2000;133:55-65. [PubMed: 10933210]

Kringelbach ML. The human orbitofrontal cortex: linking reward to hedonic experience. Nat. Neurosci 2005;6:691-702.

Kringelbach ML, Rolls ET. The functional neuroanatomy of the human orbitofrontal cortex: evidence from neuroimaging and neuropsychology. Prog. Neurobiol 2004;72:341-72. [PubMed: 15157726]

Kunda Z, Thagard P. Forming impressions from stereotypes, traits, and behaviors: a parallel-constraintsatisfaction theory. Psychol. Rev 1996;103:284-308.

Lacerda ALT, Keshavan MS, Hardan AY, Yorbik O, Brambilla P, et al. Anatomic evaluation of the orbitofrontal cortex in major depressive disorder. Biol. Psychiatry 2004;55:353-58. [PubMed: 14960287]

Lambie JA, Marcel AJ. Consciousness and the varieties of emotion experience: a theoretical framework. Psychol. Rev 2002;109:219-59. [PubMed: 11990318]

Lane RD, Ahern GL, Schwartz GE, Kaszniak AW. Is alexithymia the emotional equivalent of blindsight? Biol. Psychiatry 1997;42:834-44. [PubMed: 9347133]

Lane RD, Garfield DAS. Becoming aware of feelings: integration of cognitive-developmental, neuroscientific, and psychoanalytic perspectives. Neuropsychoanalysis 2005;7:1-66.

Lane, RD.; McRae, K. Neural substrates of conscious emotional experience: a cognitive-neuroscientific perspective. In: Beauregard, M., editor. Consciousness, Emotional Self-Regulation and the Brain. Benjamins; Amsterdam: 2004. p. 87-122.

Lane RD, Schwartz GE. Levels of emotional awareness: a cognitive-developmental theory and its application to psychopathology. Am. J. Psychiatry 1987;144:133-43. [PubMed: 3812780]

Lang P, Greenwald M, Bradley M, Hamm A. Looking at pictures: affective, facial, visceral, and behavioral reactions. Psychophysiology 1993;30:261-73. [PubMed: 8497555]

Lazarus, RS. Psychological Stress and the Coping Process. McGraw-Hill; New York: 1966.

Lazarus, RS. Emotion and Adaptation. Oxford Univ. Press; New York: 1991.

Lazarus, RS.; Folkman, S. Stress, Appraisal and Coping. Springer; New York: 1984.

LeDoux, JE. The Emotional Brain: The Mysterious Underpinnings of Emotional Life. Simon \& Schuster; New York: 1996.

LeDoux JE. Emotion circuits in the brain. Annu. Rev. Neurosci 2000;23:155-84. [PubMed: 10845062]

Leu J, Mesquita B, Ellsworth PC, Yong ZZ, Huijian Y, et al. Cultural models of emotion regulation in East and West: "dialectical" emotions vs. the pursuit of pleasantness. 2006Manuscr. under review

Leventhal H, Scherer K. The relationship of emotion to cognition: a functional approach to a semantic controversy. Cogn. Emot 1987;1:3-28.

Lieberman, MD.; Gaunt, R.; Gilbert, DT.; Trope, Y. Reflexion and reflection: a social cognitive neuroscience approach to attributional inference. In: Zanna, MP., editor. Advances in Experimental Social Psychology. 34. Academic; San Diego: 2002. p. 199-49.

Llinas R, Ribary U, Contreras D, Pedroarena C. The neuronal basis for consciousness. Philos. Trans. R. Soc. Lond. B Biol. Sci 1998;353:1841-49. [PubMed: 9854256]

Loewenstein, G.; Lerner, JS. The role of affect in decision making. In: Goldsmith, H.; Davidson, R.; Scherer, K., editors. Handbook of Affective Science. Oxford Univ. Press; New York: 2003. p. 619-42. 
Maddock RJ. The retrosplenial cortex and emotion: new insights from functional neuroimaging of the human brain. Trends Neurosci 1999;22:310-16. [PubMed: 10370255]

Maia TV, Cleeremans A. Consciousness: converging insights from connectionist modeling and neuroscience. Trends Cogn. Sci 2005;9:397-404. [PubMed: 16005677]

Mantani T, Okamoto Y, Shirao N, Okada G, Yamawaki S. Reduced activation of posterior cingulate cortex during imagery in subjects with high degrees of alexithymia: a functional magnetic resonance imaging study. Biol. Psychiatry 2005;57:982-90. [PubMed: 15860338]

Markus HR, Kitayama S. Culture and the self: implications for cognition, emotion, and motivation. Psychol. Rev 1991;98:224-53.

Markus, HR.; Kitayama, S. The cultural construction of self and emotion: implications for social behavior. In: Kitayama, S.; Markus, HR., editors. Emotion and Culture: Empirical Studies of Mutual Influence. Am. Psychol. Assoc.; Washington, DC: 1994. p. 89-130.

Martin A, Chao LL. Semantic memory and the brain: structure and processes. Curr. Opin. Neurobiol 2001;11:194-201. [PubMed: 11301239]

Mauro R, Sato K, Tucker J. The role of appraisal in human emotions: a cross-cultural study. J. Personal. Soc. Psychol 1992;62:301-17.

Mayberg HS. Limbic-cortical dysregulation: a proposed model of depression. J. Neuropsychiatry Clin. Neurosci 1997;9:471-81. [PubMed: 9276848]

Mayer JD, Gaschke YN. The experience and meta-experience of mood. J. Personal. Soc. Psychol 1988;55:102-11.

McDonald AJ. Cortical pathways to the mammalian amygdala. Prog. Neurobiol 1998;55:257-332. [PubMed: 9643556]

Mesquita B. Emotions in collectivist and individualist contexts. J. Personal. Soc. Psychol 2001;80(1): 68-74.

Mesquita, B. Emotions as dynamic cultural phenomena. In: Goldsmith, H.; Davidson, R.; Scherer, K., editors. Handbook of the Affective Sciences. Oxford; New York: 2003. p. 871-90.

Mesquita, B.; Ellsworth, PC. The role of culture in appraisal. In: Sherer, KR.; Schorr, SA., editors. Appraisal Processes in Emotion: Theory, Methods, Research. Oxford Univ. Press; New York: 2001. p. 233-48.

Mesquita B, Frijda NH. Cultural variations in emotions: a review. Psychol. Bull 1992;112:179-204. [PubMed: 1454891]

Mesquita, B.; Leu, J. The cultural psychology of emotion. In: Cohen, SKD., editor. Handbook of Cultural Psychology. Guilford; New York: 2006. In press

Mesquita B, Kawasaka M. Different emotional lives. Cogn. Emot 2002;16:127-41.

Messinger DS. Positive and negative: infant facial expressions and emotions. Curr. Dir. Psychol. Sci 2002;11:1-6.

Mesulam, MM. Principles of Behavioral and Cognitive Neurology. Oxford Univ. Press; New York: 2000.

Milad MR, Orr SP, Pitman RK, Rauch SL. Context modulation of memory for fear extinction in humans. Psychophysiology 2005;42:456-64. [PubMed: 16008774]

Mitchell JP, Banaji MR, Macrae CN. The link between social cognition and self-referential thought in the medial prefrontal cortex. J. Cogn. Neurosci 2005a;17:1306-15. [PubMed: 16197685]

Mitchell JP, Banaji MR, Macrae CN. General and specific contributions of the medial prefrontal cortex to knowledge about mental states. NeuroImage 2005b;28:757-62. [PubMed: 16325141]

Mitchell JP, Macrae CN, Banaji MR. Forming impressions of people versus inanimate objects: socialcognitive processing in the medial prefrontal cortex. NeuroImage 2005c;26:251-57. [PubMed: 15862225]

Morris JS, Dolan RJ. Dissociable amygdala and orbitofrontal responses during reversal fear conditioning. Neuroimage 2004;22:372-80. [PubMed: 15110029]

Moses EB, Barlow DH. A new unified treatment approach for emotional disorders based on emotion science. Curr. Dir. Psychol. Sci. 2006In press

Murphy FC, Nimmo-Smith I, Lawrence AD. Functional neuroanatomy of emotions: a meta-analysis. Cogn. Affect. Behav. Neurosci 2003;3:207-33. [PubMed: 14672157]

Nagel, E. The Structure of Science. Routledge \& Kegan Paul; London: 1961. 
Naghavi HR, Nyberg L. Common fronto-parietal activity in attention, memory, and consciousness: shared demands on integration? Conscious. Cogn 2005;14:390-425. [PubMed: 15950889]

Nakao T, Nakagawa A, Yoshiura T, Nakatani E, Nabeyama M, et al. Brain activation of patients with obsessive-compulsive disorder during neuropsychological and symptom provocation tasks before and after symptom improvement: a functional magnetic resonance imaging study. Biol. Psychiatry 2005;57:901-10. [PubMed: 15820711]

Nauta WJ. The problem of the frontal lobe: a reinterpretation. J. Psychiatric Res 1971;8:167-87.

Niedenthal PM, Barsalou LW, Winkielman P, Krauth-Gruber S, Ric F. Embodiment in attitudes, social perception, and emotion. Personal. Soc. Psychol. Rev 2005;9:184-211.

Nisbett RE, Wilson TD. Telling more than we can know: verbal reports on mental processes. Psychol. Rev 1977;84:231-59.

Oatley K, Johnson-Laird PN. Towards a cognitive theory of emotions. Cogn. Emot 1987;1:29-50.

Ochsner KN, Beer JS, Robertson ER, Cooper JC, Gabrieli JDE, et al. The neural correlates of direct and reflected self-knowledge. NeuroImage 2005;28:797-814. [PubMed: 16290016]

Ochsner KN, Gross JJ. The cognitive control of emotion. Trends Cogn. Sci 2005;9:242-49. [PubMed: 15866151]

Ochsner, KN.; Gross, JJ. The functional architecture of emotion regulation. In: Gross, JJ., editor. The Handbook of Emotion Regulation. Guilford; New York: 2007. In press

Ochsner KN, Knierim K, Ludlow DH, Hanelin J, Ramachandran T, et al. Reflecting upon feelings: an fMRI study of neural systems supporting the attribution of emotion to self and other. J. Cogn. Neurosci 2004;16:1746-72. [PubMed: 15701226]

Ongur D, Ferry AT, Price JL. Architectonic subdivision of the human orbital and medial prefrontal cortex. J. Comp. Neurol 2003;460:425-49. [PubMed: 12692859]

Ongur D, Price JL. The organization of networks within the orbital and medial prefrontal cortex of rats, monkeys and humans. Cereb. Cortex 2000;10:206-19. [PubMed: 10731217]

Ortony A, Turner TJ. What's basic about basic emotions? Psychol. Rev 1990;97:315-31. [PubMed: 1669960]

Panksepp, J. Affective Neuroscience: The Foundations of Human and Animal Emotions. Oxford Univ. Press; London: 1998.

Parkinson B. Untangling the appraisal-emotion connection. Personal. Soc. Psychol. Rev 1997;1:62-79.

Parvizi J, Damasio A. Consciousness and the brainstem. Cognition 2001;79:135-59. [PubMed: 11164026]

Pezawas L, Meyer-Lindenberg A, Drabant EM, Verchinski BA, Munoz KE, et al. 5-HTTLPR polymorphism impacts human cingulate-amygdala interactions: a genetic susceptibility mechanism for depression. Nat. Neurosci 2005;8:828-34. [PubMed: 15880108]

Phan KL, Wager T, Taylor SF, Liberzon I. Functional neuroanatomy of emotion: a meta-analysis of emotion activation studies in PET and fMRI. Neuroimage 2002;16:331-48. [PubMed: 12030820]

Phelps EA. Emotion and cognition: insights from studies of the human amygdala. Annu. Rev. Psychol 2006;57:27-53. [PubMed: 16318588]

Phelps EA, Delgado MR, Nearing KI, LeDoux JE. Extinction learning in humans: role of the amygdala and vmPFC. Neuron 2004;43:897-905. [PubMed: 15363399]

Phillips ML, Drevets WC, Rauch SL, Lane R. Neurobiology of emotion perception I: the neural basis of normal emotion perception. Biol. Psychiatry 2003;54:504-14. [PubMed: 12946879]

Plutchik, R. Emotion: A Psychoevolutionary Synthesis. Harper \& Row; New York: 1980.

Poldrack RA, Wagner AD, Prull MW, Desmond JE, Glover GH, Gabrieli JD. Functional specialization for semantic and phonological processing in the left inferior prefrontal cortex. Neuroimage 1999;10:15-35. [PubMed: 10385578]

Pollatos O, Kirsch W, Schandry R. On the relationship between interoceptive awareness, emotional experience, and brain processes. Cogn. Brain Res 2005;25:948-62.

Power, MDT. Cognition and Emotion: From Order to Disorder. Erlbaum; Mahwah. NJ: 1997.

Pressman SD, Cohen S. Does positive affect influence health? Psychol. Bull 2005;131:925-71. [PubMed: 16351329] 
Quigley, KS.; Barrett, LF. Emotional learning and mechanisms of intentional psychological change. In: Lerner, RM.; Brandtstadter, J., editors. Action and Development: Origins and Functions of Intentional Self-Development. Sage; Thousand Oaks, CA: 1999. p. 435-64.

Quirk GJ, Russo GK, Barron JL, Lebron K. The role of ventromedial prefrontal cortex in the recovery of extinguished fear. J. Neurosci 2000;20:6225-31. [PubMed: 10934272]

Rauch SL, Whalen PJ, Shin LM, McInerney SC, Macklin ML, et al. Exaggerated amygdala response to masked facial stimuli in posttraumatic stress disorder: a functional MRI study. Biol. Psychiatry 2000;47:769-76. [PubMed: 10812035]

Reed SJ, Vanman EJ, Miller LC. Connectionism, parallel constraint satisfaction processes, and Gestalt principles: (re)introducing cognitive dynamics to social psychology. Personal. Soc. Psychol. Rev 1997;1:26-53.

Robinson M, Clore GL. Episodic and semantic knowledge in emotional self-report: evidence for two judgment processes. J. Personal. Soc. Psychol 2002;83:198-215.

Roseman IJ. Appraisal determinants of discrete emotions. Cogn. Emot 1991;5:161-200.

Roseman IJ, Antoniou AA, Jose PE. Appraisal determinants of emotions: constructing a more accurate and comprehensive theory. Cogn. Emot 1996;10:241-77.

Roseman IJ, Spindel MS, Jose PE. Appraisals of emotion-eliciting events: testing a theory of discrete emotions. J. Personal. Soc. Psychol 1990;59:899-915.

Rothbaum F, Pott M, Azuma H, Miyake K, Weisz J. The development of close relationships in Japan and the United States: paths of symbiotic harmony and generative tension. Child Dev 2000;71:1121-42. [PubMed: 11108082]

Russell JA. A circumplex model of affect. J. Personal. Soc. Psychol 1980;39:1161-78.

Russell JA. Pancultural aspects of human conceptual organization of emotions. J. Personal. Soc. Psychol 1983;45:1281-88.

Russell JA. Core affect and the psychological construction of emotion. Psychol. Rev 2003;110:145-72. [PubMed: 12529060]

Russell JA, Barrett LF. Core affect, prototypical emotional episodes, and other things called emotion: dissecting the elephant. J. Personal. Soc. Psychol 1999;76:805-19.

Russell JA, Mehrabian A. Evidence for a three-factor theory of emotions. J. Res. Personal 1977;11:27394.

Russell JA, Weiss A, Mendelsohn GA. Affect grid: a single-item scale of pleasure and arousal. J. Personal. Soc. Psychol 1989;57:493-502.

Ryle, G. The Concept of Mind. Univ. Chicago Press; Chicago, IL: 2000.

Sabini J, Silver M. Why emotion names and experiences don't neatly pair. Psychol. Inq 2005;16:1-10.

Salamone JD, Correa M. Motivational views of reinforcement: implications for understanding the behavioral functions of nucleus accumbens dopamine. Behav. Brain Res 2002;137:3-25. [PubMed: 12445713]

Salamone JD, Correa M, Mingote SM, Weber SM. Beyond the reward hypothesis: alternative functions of nucleus accumbens dopamine. Curr. Opin. Pharmacol 2005;5:34-41. [PubMed: 15661623]

Salamone JD, Correa M, Mingote SM, Weber SM, Farrar AM. Nucleus accumbens dopamine and the forebrain circuitry involved in behavioral activation and effort-related decision making: implications for understanding anergia and psychomotor slowing in depression. Curr. Psychiatry Rev. 2006In press

Schachter S, Singer JE. Cognitive, social, and physiological determinants of emotional state. Psychol. Rev 1962;69:379-99. [PubMed: 14497895] errata 121

Scherer, KR. On the nature and function of emotion: a component process approach. In: Scherer, KR.; Ekman, P., editors. Approaches to Emotion. Erlbaum; Hillsdale, NJ: 1984. p. 293-317.

Scherer KR. Profiles of emotion-antecedent appraisal: testing theoretical predictions across cultures. Cogn. Emot 1997a;11:113-50.

Scherer KR. The role of culture in emotion-antecedent appraisal. J. Personal. Soc. Psychol 1997b;73:90222. 
Schimmack U, Oishi S, Diener E. Cultural influences on the relation between pleasant emotions and unpleasant emotions: Asian dialectic philosophies or individualism-collectivism? Cogn. Emot 2002;19:705-19.

Schnider A, Treyer V, Buck A. Selection of currently relevant memories by the human posterior medial orbitofrontal cortex. J. Neurosci 2000;20:5880-84. [PubMed: 10908632]

Schnyer DM, Nicholls L, Verfaellie M. The role of VMPC in metamemorial judgments of content retrievability. J. Cogn. Neurosci 2005;17:832-46. [PubMed: 15904549]

Schultz W. Neural coding of basic reward terms of animal learning theory, game theory, microeconomics and behavioural ecology. Curr. Opin. Neurobiol 2004;14:139-47. [PubMed: 15082317]

Schultz W, Tremblay L, Hollerman JR. Reward processing in primate orbitofrontal cortex and basal ganglia. Cereb. Cortex 2000;10:272-84. [PubMed: 10731222]

Schwarz N, Clore GL. Mood, misattribution, and judgments of well-being: informative and directive functions of affective states. J. Personal. Soc. Psychol 1983;45:513-23.

Scollon CN, Diener E, Oishi S, Biswas-Diener R. An experience sampling and cross-cultural investigation of the relation between pleasant and unpleasant affect. Cogn. Emot 2005;19:27-52.

Searle, JR. The Rediscovery of the Mind. MIT Press; Cambridge, MA: 1992.

Searle JR. Consciousness. Annu. Rev. Neurosci 2000;23:557-78. [PubMed: 10845075]

Searle, JR. Mind. Oxford Univ. Press; New York: 2004.

Shamay-Tsoory SG, Lester H, Chisin R, Israel O, Bar-Shalom R, et al. The neural correlates of understanding the other's distress: a positron emission tomography investigation of accurate empathy. Neuroimage 2005;27:468-72. [PubMed: 15987670]

Shamay-Tsoory SG, Tomer R, Berger BD, Aharon-Peretz J. Characterization of empathy deficits following prefrontal brain damage: the role of the right ventromedial prefrontal cortex. J. Cogn. Neurosci 2003;15:324-37. [PubMed: 12729486]

Shamay-Tsoory SG, Tomer R, Goldsher D, Berger BD, Aharon-Peretz J. Impairment in cognitive and affective empathy in patients with brain lesions: anatomical and cognitive correlates. J. Clin. Exp. Neuropsychol 2004;26:1113-27. [PubMed: 15590464]

Shaver P, Schwartz J, Kirson D, O'Connor C. Emotion knowledge: further exploration of a prototype approach. J. Personal. Soc. Psychol 1987;52:1061-86.

Shin LM, Orr SP, Carson MA, Rauch SL, Macklin ML, et al. Regional cerebral blood flow in the amygdala and medial prefrontal cortex during traumatic imagery in male and female Vietnam veterans with PTSD. Arch. Gen. Psychiatry 2004;61:168-76. [PubMed: 14757593]

Shin LM, Wright CI, Cannistraro PA, Wedig MM, McMullin K, et al. A functional magnetic resonance imaging study of amygdala and medial prefrontal cortex responses to overtly presented fearful faces in posttraumatic stress disorder. Arch. Gen. Psychiatry 2005;62:273-81. [PubMed: 15753240]

Shweder, RA. The cultural psychology of emotions. In: Lewis, M.; Haviland-Jones, M., editors. Handbook of Emotion. Guilford; New York: 1993. p. 417-31.

Shweder, RA.; Haidt, J. The cultural psychology of emotions: ancient and new. In: Lewis, M.; HavilandJones, M., editors. Handbook of Emotions. Guilford; New York: 2000. p. 397-414.

Skinner, BF. Science and Human Behavior. Macmillan; New York: 1953.

Smith CA, Ellsworth PC. Patterns of cognitive appraisal in emotion. J. Personal. Soc. Psychol 1985;48:813-38.

Smith CA, Ellsworth PC. Patterns of appraisal and emotion related to taking an exam. J. Personal. Soc. Psychol 1987;52:475-88.

Smith ER, DeCoster J. Dual-process models in social and cognitive psychology: conceptual integration and links to underlying memory systems. Personal. Soc. Psychol. Rev 2000;4:108-31.

Spelke ES. Core knowledge. Am. Psychol 2000;55:1233-43. [PubMed: 11280937]

Spitz, RA. The First Year of Life. Int. Univ. Press; New York: 1965.

Sroufe, LA. Socioemotional development. In: Osofsky, JD., editor. Handbook of Infant Development. Wiley; New York: 1979. p. 462-516.

Stefanacci L, Amaral DG. Some observations on cortical inputs to the macaque monkey amygdala: an anterograde tracing study. J. Comp. Neurol 2002;451:301-23. [PubMed: 12210126] 
Stein DJ, Westenberg HGM, Liebowitz MR. Social anxiety disorder and generalized anxiety disorder: serotonergic and dopaminergic neurocircuitry. J. Clin. Psychiatry 2002;63:12-19. [PubMed: 12027115]

Talairach, J.; Tournoux, P. Co-Planar Stereotaxic Atlas of the Human Brain. Thieme; Stuttgart: 1988.

Valente AAJ, Miguel EC, Castro CC, Amaro EJ, Duran FLS, et al. Regional gray matter abnormalities in obsessive-compulsive disorder: a voxel-based morphometry study. Biol. Psychiatry 2005;58:479-87. [PubMed: 15978549]

Wager TD, Phan KL, Liberzon I, Taylor SF. Valence, gender, and lateralization of functional brain anatomy in emotion: a meta-analysis of findings from neuroimaging. NeuroImage 2003;19:51331. [PubMed: 12880784]

Wagner AD, Maril A, Bjork RA, Schacter DL. Prefrontal contributions to executive control: fMRI evidence for functional distinctions within lateral prefrontal cortex. Neuroimage 2001;14:1337-47. [PubMed: 11707089]

Watson, D.; Clark, LA. Univ. Iowa; Iowa City: 1994. Manual for the positive and negative affect schedule (expanded form). Unpubl. manuscr.

Watson D, Tellegen A. Toward a consensual structure of mood. Psychol. Bull 1985;98:219-35. [PubMed: 3901060]

Watson D, Wiese D, Vaidya J, Tellegen A. The two general activation systems of affect: structural findings, evolutionary considerations, and psychobiological evidence. J. Personal. Soc. Psychol 1999;76:820-38.

Watson JB. A schematic outline of the emotions. Psychol. Rev 1919;26:165-96.

Weinstock LM, Whisman MA. Neuroticism as a common feature of the depressive and anxiety disorders: a test of the Revised Integration Hierarchical Model in a national sample. J. Abnorm. Psychol 2006;115:68-74. [PubMed: 16492097]

Weniger G, Irle E. Impaired facial affect recognition and emotional changes in subjects with transmodal cortical lesions. Cereb. Cortex 2002;12:258-68. [PubMed: 11839600]

Wiens S. Interoception in emotional experience. Curr. Opin. Neurol 2005;18:442-47. [PubMed: 16003122]

Wilson, D.; Sperber, D. Relevance theory. In: Ward, G.; Horn, L., editors. Handbook of Pragmatics. Blackwell Sci.; Oxford, UK: 2003. p. 607-32.

Wilson TD, Dunn EW. Self-knowledge: its limits, value and potential for improvement. Annu. Rev. Psychol 2004;55:493-518. [PubMed: 14744224]

Winkielman P, Berridge KC, Wilbarger JL. 2005:335-62.See Barrett et al. 2005

Wundt, WM. Outlines of Psychology. Engelmann; Leipzig: 1897.

Yamasaki H, LaBar KS, McCarthy G. Dissociable prefrontal brain systems for attention and emotion. Proc. Natl. Acad. Sci. USA 2002;99:11447-51. [PubMed: 12177452]

Yik M. Culture, gender, and the bipolarity of affect. Cogn. Emot. 2006In press

Yik MSM, Russell JA, Barrett LF. Structure of self-reported current affect: integration and beyond. J. Personal. Soc. Psychol 1999;77:600-19.

Zeki S. The disunity of consciousness. Trends Cogn. Sci 2003;7:214-18. [PubMed: 12757823]

Zuckerman, M.; Lubin, B. Manual for the MAACL-R: The Multiple Affect Adjective Checklist Revised. Educ. Ind. Test. Serv.; San Diego, CA: 1985. 


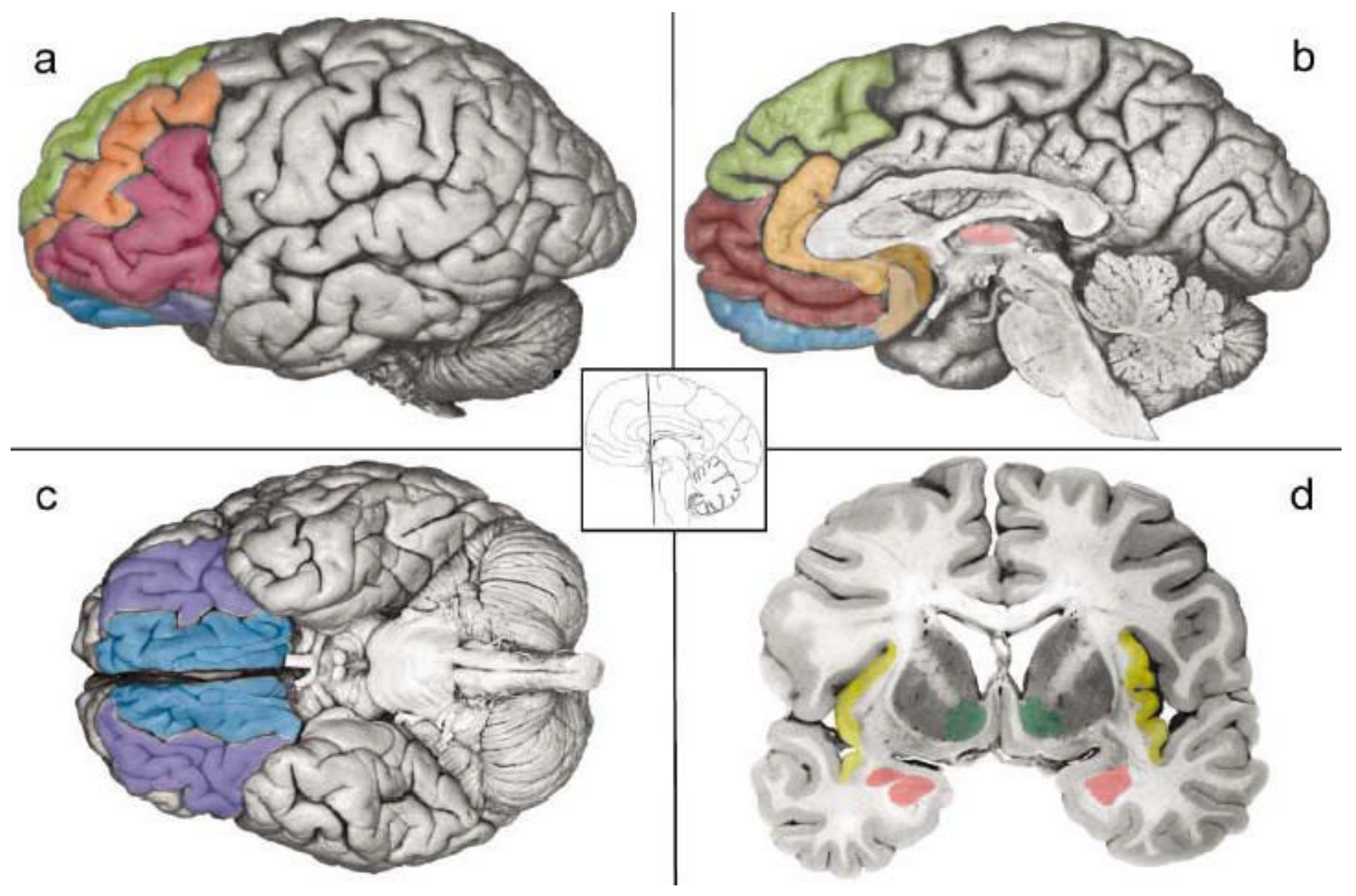

Figure 1.

Key brain areas in the neural reference space for mental representations of emotion. The ventral system for core affect includes two closely connected circuits that are anchored in the orbitofrontal cortex (the entire ventral surface of the front part of the brain lying behind the orbital bone above the eye; Figure 1c). The sensory system involves the lateral sector of the orbitofrontal cortex (OFC) and includes the lateral portions of BA 11 and 13, BA 47/12 (a,c, purple). It is closely connected to the anterior insula ( $d$, yellow) and the basolateral (BL) complex in the amygdala ( $d$, rose, ventral aspect). The visceromotor circuitry includes the ventral portion of the ventromedial prefrontal cortex (VMPFC), which lies in the medial sector of the $\operatorname{OFC}(a, b, c, b l u e)$ and includes medial BA 11 and 13 ventral portions of BA 10 , as well as BA 14, where the medial and lateral aspects of OFC connect; VMPFC is closely connected to the amygdala (including the central nucleus, $d$, rose, dorsal aspect) and the subgenual parts of the anterior cingulate cortex involving the anterior aspects of BA 24, 25, and 32 on the medial wall of the brain (ACC; $b$, copper and tan). The dorsal system is associated with mental state attributions including the dorsal aspect of the VMPFC corresponding to the frontal pole in BA 10 (b, maroon), the anterior ACC (peach), and the dorsomedial prefrontal cortex

(DMPFC) corresponding to the medial aspects of BA 8, 9, and $10(a, b$, green). Ventrolateral prefrontal cortex (VLPFC) is shown in red (a). Also shown for reference are the thalamus $(b$, light pink), the ventral striatum ( $d$, green), and the middle frontal gyrus in the dorsolateral prefrontal cortex (a, orange). Photographs adapted from DeArmond et al. (1989, pp. 5, 7, 8, and 43). 


\section{a}

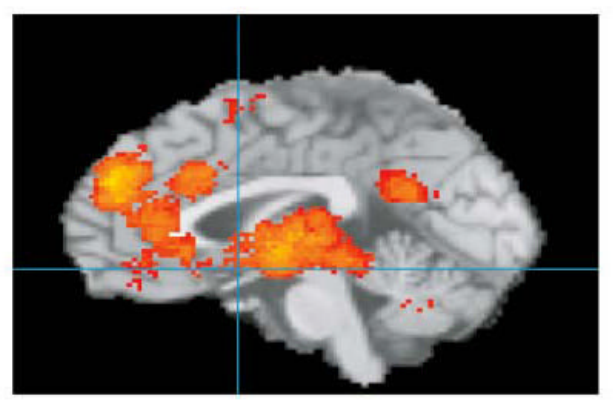

b

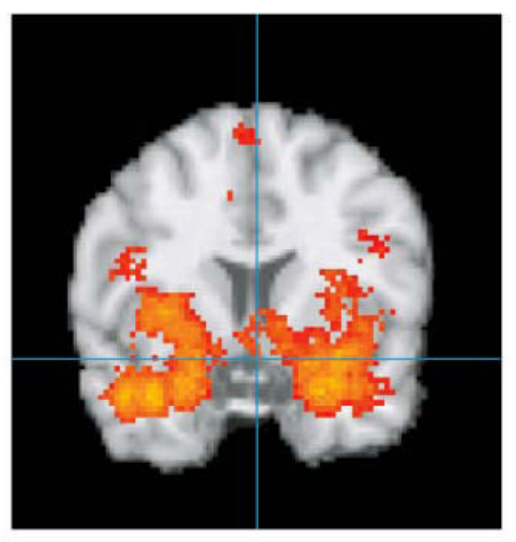

C

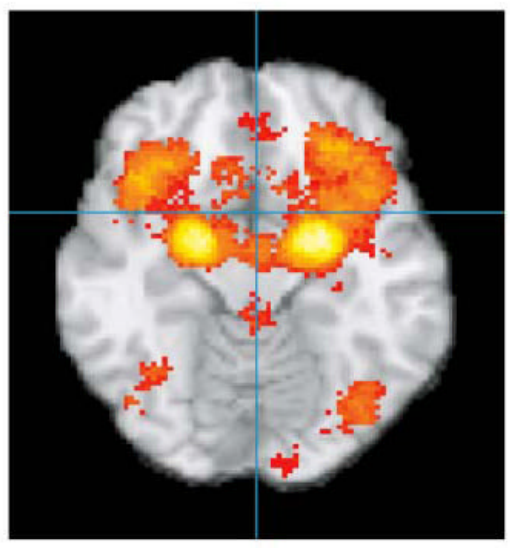

Figure 2.

Preliminary summary of neuroimaging studies of core affective and emotion experiences. Activation foci were registered to a common stereotaxic brain atlas (Talairach \& Tournoux 1988) where $\mathrm{x}=$ distance in millimeters to the right $(+)$ or left $(-)$ of midline; $\mathrm{y}=$ distance anterior $(+)$ or posterior $(-)$ to the anterior commissure; and $\mathrm{z}=$ distance superior $(+)$ or inferior $(-)$ to a horizontal plane through the anterior and posterior commissures. Midsagital $(a, \mathrm{x}=0)$, coronal $(b, \mathrm{y}=7)$, and horizontal $(c, \mathrm{z}=-13)$ images are presented. Significant areas of activation include OFC, insula, amygdala, ACC, and DMPFC (as well as VLPFC; not shown). VMPFC activations were also observed, but it is not clear that they extend down to the ventral surface, $c$, probably owing to problems with imaging that area of the brain. Lighter colors 
indicate a larger number of studies reported significant peak activations at that location (summary corrected for false discovery rate). 